CAHIERS DE

NARRATOLOGIE

\section{Cahiers de Narratologie}

Analyse et théorie narratives

32 | 2017

Récit et argumentation, interactions, lieux et dispositifs sociaux

\title{
La notion de temporalité affective confrontée à différentes approches de la narration en sciences du langage : apports, enjeux, méthodes
}

\section{Marie Carcassonne}

\section{OpenEdition}

\section{Journals}

Electronic version

URL: http://journals.openedition.org/narratologie/7866

DOI: 10.4000/narratologie.7866

ISSN: 1765-307X

Publisher

LIRCES

Electronic reference

Marie Carcassonne, « La notion de temporalité affective confrontée à différentes approches de la narration en sciences du langage : apports, enjeux, méthodes », Cahiers de Narratologie [Online], 32 | 2017, Online since 21 December 2017, connection on 19 April 2019. URL : http:// journals.openedition.org/narratologie/7866; DOI : 10.4000/narratologie.7866

This text was automatically generated on 19 April 2019

Article L.111-1 du Code de la propriété intellectuelle. 


\title{
La notion de temporalité affective confrontée à différentes approches de la narration en sciences du langage : apports, enjeux, méthodes
}

\author{
Marie Carcassonne
}

1 La notion de temporalité affective (Carcassonne 2004, 2007) insiste sur le fait que l'évocation du passé, du présent et du futur ne renvoie pas seulement à des aspects temporels chronologiques mesurables mais aussi à une dimension affective, laquelle constitue une entrée pour analyser la temporalité dans le récit. Nous allons questionner ici cette notion en la confrontant à différentes approches de la narration en sciences du langage, et en prenant le fonctionnement langagier de l'entretien biographique comme un objet d'étude en lui-même ${ }^{1}$.

2 Le corpus d'entretiens biographiques dont il sera question ici a été recueilli par des étudiants en Master 1 CCA (Contrôle Comptabilité Audit) auprès d'experts comptables (EC) travaillant en petit cabinet, dans le cadre d'un cours intitulé «communication professionnelle ». Ces étudiants ont eu à interroger des professionnels de la comptabilité sur leur activité actuelle, mais aussi sur leurs parcours ainsi que sur les conseils à donner pour suivre une voie professionnelle liée à la comptabilité2. Cet exercice pédagogique de recueil (puis d'analyse ${ }^{3}$ ) d'entretiens s'inscrit dans l'affirmation d'une certaine posture pédagogique, sur un arrière-fond de controverse opposant, pour le dire vite, les prescripteurs de l'efficacité communicationnelle aux observateurs des normes et usages langagiers en vigueur dans un milieu socio-professionnel donné. Onze entretiens ont été sélectionnés parmi une quarantaine pour l'analyse ici présentée, et quatre d'entre eux vont être cités en tant qu'entretiens représentatifs.

3 Nous présenterons dans un premier temps (I) la notion de temporalité affective après avoir précisé la perspective dialogique dans laquelle nous nous inscrivons.

4 Nous verrons ensuite (II) en quoi les approches structuralistes du récit ont insisté sur une forme de temporalité que l'on peut caractériser comme tendue vers la fin du récit (ce qui 
crée un certain suspense); puis (III) nous aborderons l'approche herméneutique de Ricœur qui a insisté sur une temporalité rétrospective (due aux nombreuses explications et justifications données a posteriori par le narrateur). Enfin (IV) nous examinerons en quoi certaines approches interactives et dialogiques du récit, qui tiennent compte des conditions de production et de réception du discours, permettent de mettre davantage en évidence les dimensions affectives de la temporalité.

5 Nous verrons que cette dimension n'avait pas échappé aux approches précédentes, qui l'avaient effleurée plus que traitée en tant que telle, préférant insister sur la temporalité que le narrateur organise plus que sur la temporalité qui l'affecte.

\section{Perspective dialogique et dimensions affectives de la temporalité}

6 C'est avant tout grâce à l'adoption d'une perspective dialogique (et surtout autodialogique, c'est-à-dire s'intéressant aux mouvements du discours de soi sur le discours de soi) que nous pourrons mettre en évidence les aspects affectifs de la temporalité dans le corpus ici présenté.

\section{Perspective dialogique}

7 La perspective dialogique introduite par Volochinov (publiée sous le nom de Bakhtine, 1977 [1929] $)^{4}$, aujourd'hui classique, a insisté sur l'idée que toute énonciation s'inscrit dans un processus ininterrompu ${ }^{5}$ de «reprises» (non nécessairement littérales) d'énoncés antérieurs mais aussi d'anticipations de réponses : un roman peut être vu, tout comme la réplique d'un dialogue, comme une réponse et comme suscitant à son tour une réponse. On peut repérer dans tout discours des énoncés "échos" renvoyant à un discours produit antérieurement (in absentia comme in praesentia) comme des énoncés « responsifs » anticipant la réponse qui va être faite au discours en question.

A la suite de Volochinov, Bakhtine a prolongé cette perspective ${ }^{6}$ et davantage insisté sur les mouvements dialogiques repérables au sein d'un « dialogue réel ».

9 En s'inspirant largement de Spitzer, Bakhtine a indiqué en quoi un énoncé repris à un autre interlocuteur dans le dialogue (même littéralement), constituait toujours une «modification» de sens. (Bakhtine, $1970: 254)$

10 Les deux auteurs russes ont ainsi souligné que la circulation des significations dans la vie sociale est liée au fait que tout locuteur reprend, modifie, et anticipe dans son discours les énoncés des autres (alterdialogisme) mais aussi les siens (autodialogisme) : même s'ils ont peut-être moins fait l'objet d'études ${ }^{7}$ par la suite, les « rapports de dialogue entre le sujet parlant et sa propre parole» (Bakhtine : 1970 [1963] [1929] : 212) font pleinement partie de la perspective dialogique au sens où l'entendaient Volochinov et Bakhtine. Un certain nombre d'auteurs ont (re)développé cette perspective dialogique en tenant compte de ces deux versants (auto et alter), entre autres François (1982), Authier-Revuz (1982), SalazarOrvig (1999), Bres (2005).

11 François a ainsi proposé au début des années 80 une « approche dialogique du dialogue " (1984 : 167) comme du récit ${ }^{8}$, en insistant sur le déplacement produit par les mouvements de reprise et/ou de modifications des propos antérieurs, qu'il s'agisse de ceux de l'autre ou de soi. Il s'agissait pour lui à l'époque de mieux distinguer les approches linguistiques 
"classiques » d'une telle approche «dialogique » (ou «langagière ») $)^{9}$. Authier-Revuz a suggéré quant à elle le terme d'" autodialogisme » (1982, 1995 : 148-160) pour renvoyer aux mouvements discursifs de soi sur soi, tandis que Bres (2005) a préféré plus récemment adopter l'expression de « dialogisme intralocutif »:

Le locuteur est son premier interlocuteur [...] dans le processus de l'autoréception :

la production de sa parole se fait constamment en interaction avec ce qu'il a dit

antérieurement, avec ce qu'il est en train de dire, et avec ce qu'il a à dire (2005:52).

Bres (2005) a plus globalement proposé de relire Volochinov et Bakhtine en distinguant (i) le dialogisme concernant des énoncés repris et rapportables à des tiers absents (dialogisme interdiscursif), (ii) le dialogisme concernant des énoncés (repris ou anticipés) et rapportables à l'interlocuteur (dialogisme interlocutif) ou au locuteur lui-même (dialogisme intralocutif).

Notons qu'une place importante est accordée au récepteur-analyste dans une approche dialogique : en mettant en évidence certains rapports entre les paroles d'un locuteur au sein d'un même discours, ou entre ce discours et d'autres tenus ailleurs, il fait entendre sa propre voix. Tout en analysant celles des autres, il propose la sienne dans l'échange qu'il analyse (Carcassonne 2011).

a dimension autodialogique de l'interprétation sera importante pour l'analyse présentée : comme on va le voir, ce sont surtout les mouvements du discours de soi sur le discours de $s i^{10}$ qui vont ici permettre d'éclairer certains aspects de la temporalité affective: la mise en rapport de certains extraits avec d'autres extraits du même narrateur montrera en effet des déplacements de sens concernant cette temporalité affective.

\section{Dimensions affectives de la temporalité}

15 La temporalité a été définie dans différents champs (physique, mathématique, psychologique, philosophique, sociologique, linguistique) ${ }^{11}$ et les liens entre les définitions proposées par chacun de ces champs sont encore aujourd'hui sources d'interrogations : par exemple, aux philosophes qui conçoivent la temporalité comme liée à la conscience, les physiciens posent la question de savoir comment le temps a pu s'écouler avant l'apparition de l'homme sur terre, ou même avant l'apparition de la terre elle-même. Les différents champs font travailler la notion de temporalité, ce qui amène à souligner la multiplicité des temporalités sans vraiment pourvoir circonscrire la notion une fois pour toute.

16 Tout au plus peut-on préciser ici le sens où nous l'appréhendons, en indiquant les trois grands champs (philosophie, sémantique, analyse textuelle) qui nous ont inspirée car ils ont abordé, sous des angles différents, la notion de temporalité affective.

17 Nous allons d'abord indiquer rapidement ce que nous retenons de certains des auteurs appartenant à ces trois champs, puis nous circonscrirons la façon dont nous l'envisageons dans le cadre d'une analyse du discours.

\section{Des dimensions abordées dans (au moins) trois champs théoriques}

18 En philosophie, nous retenons les auteurs qui relient la temporalité à la conscience d'un sujet donné (Saint Augustin, Bergson, Husserl, etc.), ce qui implique de distinguer (i) à la suite de Bergson le temps qualitatif (durée intérieure) et quantitatif (mesurable par des 
chronomètres), mais aussi (ii) à la suite de Berger le temps opératoire (de l'action) et existentiel (à tonalité affective). Au-delà de ces premières distinctions, la perspective phénoménologique ${ }^{12}$ a plus précisément interrogé le lien entre temporalité et constitution de soi et souligné que le temps atteint la conscience par «l'affection»; il est donné au sujet d'une façon "passive » qui « l'affecte ». C'est dans ce cadre phénoménologique que s'inscrivent les "existentiaux" mis en évidence par Heidegger (1927): en tant que manières d'être au monde, ils renvoient à des « dispositions affectives » (par exemple la tristesse, la colère, l'espoir) ; c'est aussi dans ce cadre phénoménologique que MerleauPonty (1947), - qui s'inspire de Husserl (1992 [1905]) -, décrit le «flux temporel » comme une dimension de notre être et non comme un objet de savoir: la relation du sujet incarné au monde n'est pas de l'ordre de du conçu, mais relève de l'immersion

On peut néanmoins essayer selon nous de revenir réflexivement sur cette immersion. Notons ici que ce que disent les phénoménologues à propos de la temporalité vécue concerne plutôt l'interaction complexe de la temporalité vécue et de la temporalité dite : le temps dit n'est pas un simple reflet du temps vécu mais il n'est pas non plus totalement reconstruit et sans rapport avec lui. S'intéresser (comme nous proposons de le faire ici) à la « mise en mots » (François, 2004 : 11) aboutit à situer l'analyse dans un « entre-deux » : le dire ne (re)construit pas l'objet de but en blanc, mais il n'y a pas non plus un «à dire » qui préexisterait au dit.

En sémantique, un certain nombre de recherches ont souligné la dimension temporelle et/ ou spatiale des termes affectifs et les ont distingués par leur durée : les plus durables sont proches des affects « catégoriels » mis en évidence par Darwin (1872) : bonheur, tristesse, crainte, colère, dégoût, surprise, intérêt, honte (lesquels sont eux-mêmes proches des existentiaux de Heidegger) ; les moins durables correspondent à des changements d'états, de motivation, ou encore de tension ${ }^{13}$, et peuvent ponctuellement s'associer aux affects durables. Greimas et Fontanille ont par exemple souligné la dimension temporelle des affects catégoriels : « La "rancune" est un "ressentiment durable", la "patience" est une "capacité à endurer", l'"espoir" le fait "d'attendre quelque chose avec patience"; un coléreux est dit "prompt à se mettre en colère" " (1991: 78). Augustyn et Grossmann (2009) ont en outre pointé le fait que ces affects catégoriels étaient souvent associés dans le langage courant à une qualification ou à des métaphores temporelles ou spatiales précisant un changement d'état, comme dans les expressions suivantes: "joie profonde ", «immense tristesse ", «il nage dans la joie ", «la colère le submerge ", «l'angoisse monte»: les verbes "nager», «submerger», «monter» mettent tous en évidence l'idée que les affects «occupent un certain volume dans l'espace, et s'y déploient » de façon dynamique.

21 En analyse textuelle, Bakhtine (1984 [1936-38]) a différencié quatre types «concrets » (n'existant pas a priori) de romans (de voyage, d'épreuve, autobiographique, de formation) à partir d'une différenciation des relations entre le héros et les chronotopes, lesquels peuvent se définir comme l'association dans une même enveloppe discursive d'aspects spatiaux, temporels, et affectifs ou idéologiques. Le chronotope est pour lui une sorte de «matérialisation du temps dans l'espace » qui concrétise également des aspects affectifs (sentiments) et idéologiques ("généralisations philosophiques et sociales, idées, analyses des causes et des effets », Bakhtine $1978: 391$ ), ce qui lui donne une signification « figurative »: "En art et en littérature toutes les définitions spatio-temporelles sont inséparables les unes des autres, et comportent toujours une valeur émotionnelle.» (Bakhtine 1978 : 384). Il indique par exemple que dans le roman grec, l'amour « demeure 
absolu et invariable tout au long du roman » (1978 : 242). Il caractérise le chronotope de la route comme rassemblant des personnes habituellement séparées par la hiérarchie sociale ou par l'espace. Tandis qu'à l'inverse celui du salon (littéraire) est celui de la « rencontre non fortuite dans un monde connu où la hiérarchie sociale est forte et où se concrétise visiblement le pouvoir lié à l'argent" (Ibid.: 384). Ajoutons que selon Bakhtine, les interrelations entre chronotopes dans le roman sont presque toujours dialogiques: les chronotopes peuvent s'imbriquer, coexister, s'entrelacer, se succéder, se juxtaposer, s'opposer ou se trouver dans des relations réciproques plus compliquées (un chronotope pouvant par exemple dominer les autres à certains moments); et les chronotopes dans lesquels évolue un récepteur en influencent sa perception : ainsi un dialogue « entre dans le monde de l'auteur, de l'exécutant, et dans celui des auditeurs et des lecteurs, mondes chronotopiques, eux aussi » (Ibid. : 107).

Cette citation faisant intervenir le monde des lecteurs et auditeurs illustre la remarque faite au point précédent (1.1) qui soulignait le caractère éminemment réceptif d'une approche dialogique : les chronotopes se modifient (en partie) selon les discours observés et selon les modes interprétatifs de chacun. Il en est de même et plus globalement des «temporalités affectives» dont on ne saurait donner une définition a priori. Nous allons néanmoins proposer dans le point suivant une réflexion cherchant à circonscrire les caractéristiques de la notion de temporalité affective dans une perspective d'analyse de discours.

\section{La notion de temporalité affective dans une perspective d'analyse du discours}

Nous nous inspirons en partie de la définition proposée en philosophie par les phénoménologues qui voient la temporalité comme un «temps vécu par la conscience, celui dont elle fait l'expérience $\aleph^{14}$ et qui insistent sur sa dimension affective. Nous nous distinguerons de cette approche en proposant de l'étudier en tant qu'elle est montrée dans différents entretiens biographiques, à partir de sa « mise en mots », en tenant compte du fait que les temporalités affectives vécues, dites et interprétées ne se recouvrent donc pas nécessairement.

Lors de l'évocation d'une expérience ou d'une tranche de vie, un narrateur met en mots la façon dont il se souvient avoir éprouvé la temporalité, en mentionnant (souvent) ses états affectifs (ou ressentis, émotions, manières d'être au monde) ${ }^{15}$. La " mise en mots », par le choix de certaines catégorisations et l'effectuation de certains mouvements discursifs ${ }^{16}$, contribue à la reconstruction discursive des temporalités affectives (et plus globalement à celle de toute l'expérience). Dit autrement, une temporalité affective est repérable chaque fois que le rapport au temps est dit en mentionnant des affects, modifiant ce faisant le rapport à la durée et à l'espace : ainsi certains affects temporels tels que le surgissement, l'illumination, la révélation, la surprise présentent la caractéristique d'arrêter en quelque sorte le fil du passé vers le futur. On peut les qualifier de moments esthétiques n'ayant pas de fin en soi, de «il y a »; d'autres affects temporels se présentent comme étant davantage liés à notre futur, à notre capacité de faire des projets et de calculer la conséquence de nos actes (par exemple le souci ou l'inquiétude); d'autres se présentent au contraire comme liés à notre passé et faisant pression sur notre présent (par exemple la nostalgie). Cela dit, une "classification " a priori des affects temporels est impossible à établir, tant les possibilités du langage (comme de l'expérience) sont infinis et tant les modes interprétatifs par rapport à un discours donné peuvent varier. 

amènent à voir certains aspects de la temporalité affective (ou affects temporels) : dit autrement, ces derniers ne résident pas dans la seule mise en mots mais aussi dans la rencontre entre le texte et son lecteur.

de plus qu'avant même la mise en mots, le processus même de la mémoire «enregistre " les événements vécus avec une certaine dimension affective puis les transforme. Halbwachs a souligné que «le souvenir est dans une très large mesure une reconstruction du passé à l'aide de données empruntées au présent, et préparée d'ailleurs par d'autres reconstructions faites à des époques antérieures et d'où l'image d'autrefois est sortie bien altérée » (1950:59) : par exemple, sans se souvenir de sa première rentrée au lycée, on peut avoir un souvenir général de toutes ses rentrées en classe, où les impressions (affects, atmosphères) des différentes rentrées se confondent et marquent néanmoins une certaine période temporelle. C'est ainsi que le souvenir peut être vu comme une forme d'oubli (Kundera $1993: 156)^{17}$ ou du moins de transformation du vécu, transformation retenant surtout les affects, mais aussi les modifiant.

La notion de temporalité affective au sens où nous l'entendons ayant été rapidement circonscrite, nous allons maintenant présenter la façon dont trois grandes approches narratives appréhendent la temporalité, et pointer leurs liens avec la notion de temporalité affective.

\section{Approches structuralistes du récit : insistance sur une temporalité montant en tension}

Les analyses s'inscrivant dans une perspective structuraliste ont essentiellement eu pour visée de dégager une structure ou une architecture interne, et de mettre en évidence les frontières du récit par rapport à ce qui n'est pas récit. Après les travaux du formaliste russe Propp, l'approche structuraliste du récit s'est ensuite surtout développée dans les années 60 en France ${ }^{18}$ où un certain nombre de narratologues (Barthes 1966, Todorov 1968 entre autres) ont cherché à établir les critères permettant de définir le récit, en caractérisant la temporalité comme montant en tension, ce qui s'explique par le caractère « réticent » (Baroni 2009) de la narration.

\section{Une structure quasi-identique dans une centaine de contes (Propp, 1928)}

Les pères fondateurs de l'approche structuraliste sont les formalistes russes, - et en particulier Propp, qui a publié en 1928 un ouvrage mettant en évidence une structure quasi-identique dans une centaine de contes (écrits par Afanassiev). Propp a été frappé par le fait suivant: les actions des personnages peuvent être différentes d'un conte à l'autre mais elles sont récurrentes et jouent la même «fonction » dans le déroulement de l'intrigue. Propp a dégagé 31 fonctions qui se retrouvent au moins en partie dans tous les contes, et se répartissent dans trois séquences ordonnées. Par exemple, au début des contes on trouve presque toujours un personnage en état de "manque » (âgé, malade, pauvre, malheureux en amour, etc...), manque jouant une fonction de « déclencheur » de la «fonction abstraite » : « envoi ou appel d'un héros ». 
Une fonction est ainsi une action dont le personnage-exécutant importe peu; cette action est désignée par des substantifs tels que « interdiction », « interrogation », « fuite ».

Même si Propp ne l'avait pas thématisé, ces fonctions peuvent s'analyser comme une façon de dire la dimension affective de l'expérience temporelle car elles soulignent que l'on peut être affecté par un manque, un plaisir, un déplaisir, etc. De façon proche, la notion d'actant ${ }^{19}$ chez Greimas (1966), qui s'inspire de celle de «fonction » de Propp peut être vue comme teintée d'une certaine temporalité affective. En particulier, préciser au cours d'une narration quels ont été les « adjuvants » et les « opposants » qui ont aidé ou gêné la réalisation d'une action ou d'un projet aboutit à teinter la temporalité d'une dimension affective : bienveillante et encourageante pour l'adjuvant vs malveillante et décourageante pour l'opposant.

\section{Cinq étapes du récit et montée en « tension » jusqu'à l'étape 3}

2 Todorov (1968) a plus particulièrement insisté sur les cinq étapes censées structurer tout récit, ce qui a été reformulé en termes de "schéma quinaire " par Larivaille (1974) et redéveloppé en diverses variantes architecturales par Adam (1992) :

un récit idéal commence par une situation stable qu'une force quelconque vient perturber. Il en résulte un état de déséquilibre; par l'action d'une force dirigée en sens inverse, l'équilibre est rétabli; le second équilibre est bien semblable au premier, mais les deux ne sont jamais identiques. Il y a par conséquent deux types d'épisodes dans un récit : ceux qui décrivent un état (d'équilibre ou de déséquilibre) et ceux qui décrivent le passage d'un état à l'autre (Todorov, $1968: 96)^{20}$.

Jusqu'à l'étape 3 (état de " déséquilibre » chez Todorov, de « dynamique » chez Adam), il y a une montée en tension, tandis qu'ensuite s'installe une détente progressive jusqu'au retour à un nouvel équilibre.

Un certain nombre d'auteurs (Gülich et Quasthoff 1985, Adam 1992, Revaz 1997, entre autres) ont insisté plus tard sur cette notion de tension, en tant qu'elle organise la temporalité du récit qui est ainsi «tendu » vers sa fin, dans une forme de détermination rétrograde :

Il faut que cette temporalité de base soit emportée par une tension: la détermination rétrograde qui fait qu'un récit est tendu vers sa fin ( $t+r e n)$, organisé en fonction de cette situation finale. (Adam $1992: 46)^{21}$.

Baroni a pointé plus récemment le fait que cette tension était « produite par le retard de la livraison importante d'une information concernant le passé, le présent ou le futur de l'histoire racontée » $(2009: 10)$ : pour compenser ce « caractère réticent » de la narration, le lecteur est amené à produire lui-même des « anticipations incertaines » portant sur le développement à venir de l'histoire $»^{22}$.

Précisons que si la « tension » (produite par une façon « réticente » de raconter), - en tant que dimension temporelle de la narration-, se repère très bien dans certains types de récits (et en particulier les contes russes) analysés par les narratologues structuralistes, ces derniers ont néanmoins exploré d'autres aspects. Par exemple, Genette $(1972,1983)$ s'est intéressé aux différentes possibilités narratives de la chronologie en pointant différents rapports temporels entre le je narrant et le je narré. En mobilisant une perspective énonciative (puis dialogique) sur ces questions de focalisation, les recherches de Rabatel $(1997,1998,2008)$ sur la « construction textuelle du Point De Vue (PDV) ${ }^{23}$ » ont 
ensuite éclairé le fonctionnement des récits littéraires ${ }^{24}$ et notamment la temporalité narrative (par exemple avec l'introduction de « profondeur » spatio-temporelle ») ${ }^{25}$.

\section{Le récit « héroïque » de $D$}

Dans notre corpus de 11 entretiens, un seul interviewé (D) fait le choix de se raconter sur le mode d'un héros en adoptant les critères (trop rapidement décrits ci-dessus) mis en évidence par les structuralistes. Il faut souligner que certains parcours de vie ou certaines situations se prêtent peut-être plus que d'autres au fait de se raconter sur le mode d'un héros tel qu'on le trouve dans les contes analysés par Propp, c'est-à-dire un héros pour qui on se demande s'il va arriver à surmonter les épreuves qui se présentent à lui et qui finalement y arrive. Par exemple, dans les récits analysés pas le sociolinguiste Labov (1978 [1972]), la plupart des narrateurs se racontent comme des personnages ayant surmonté un danger mortel, le plus souvent avec un certain suspense ${ }^{26}$.

Cette forme narrative parait plus surprenante dans le cas de D (cf. en annexe 4 l'extrait puis son analyse linéaire selon une grille de lecture structuraliste) qui raconte en début d'entretien son parcours étudiant et professionnel dans un récit ponctuel ${ }^{27}$ en distinguant clairement cinq étapes, en citant certains adjuvants l'ayant aidé dans son parcours, et en ménageant une certaine montée en tension en début de récit : la description de la réussite de $\mathrm{D}$ en tant que stagiaire aurait pu laisser supposer qu'il accepterait de devenir associé dans ce cabinet, et son refus (1. 20) crée un effet (relatif) de surprise, dû à l'effet « intrigant » produit par D qui ne délivre pas tout de suite l'information selon laquelle il n'était pas en accord avec les valeurs de ses supérieurs; la tension ainsi produite par le retard d'une livraison d'une information importante confère à la narration un caractère " réticent », pour reprendre le terme de Baroni (2009).

La grille de lecture structuraliste appliquée au récit de $\mathrm{D}$ aboutit à mettre l'accent sur un « manque » (valeurs de « vue à long terme » et « d'apport » absentes dans le cabinet où il a fait son stage) en tant qu'élément « déclencheur » de sa décision de créer un cabinet.

Cela dit, les « embûches » apparaissent finalement rares sur le parcours de D, ce dernier se présentant comme quelqu'un qui a "réussi » et à qui tout « réussit»: les entrées d'analyse au sens structuraliste passent finalement à côté de la dimension temporelle affective dominante de ce récit, qui est celle de la fierté (voire de la vantardise) de D vis-àvis de son parcours et de son activité. Cette temporalité affective de la fierté (nous) apparait comme la caractéristique principale de ce récit. Elle se dessine plus qu'elle ne se dit explicitement (D ne dit à aucun moment «je suis fier de moi») et elle émerge essentiellement

41 (i) de la mise en affinité d'un certain nombre de termes "affectifs » et "axiologiques » (Kerbrat-Orecchioni, 1980) ${ }^{28}$ concernant le personnage de D : « enthousiaste et jeune » (l. 2) ; « compétent» (1. 24) « beaucoup de clients... « m'appréciaient » (1. 3), « aimaient la manière dont je... » (l. 6), etc.

(ii) d'un mouvement discursif opposant deux chronotopes: on repère un premier chronotope, à savoir le cabinet (espace) où $\mathrm{D}$ a fait son stage étant jeune (période) et où il a ressenti un «manque » (ses supérieurs ne pensant qu'à l'argent dans une vue à court terme : dimension affective); puis on repère un deuxième chronotope correspondant au cabinet créé par $D$ et qu'il décrit comme a) un espace qui s'est concrètement agrandi (passant de $6 \mathrm{~m}^{2}$ à deux étages sur une des plus belles avenues de Paris), b) au cours d'une certaine période postérieure à son stage (temps), c) où il se sent désormais en adéquation 
avec ses valeurs car il «apporte» de l'aide aux client dans une vision «à long terme » (dimension affective). Notons que la notion de chronotope permet ici d'éclairer la dimension affective des "états» (de déséquilibre ou d'équilibre) décrits par les structuralistes.

En mettant en avant son indépendance, sa liberté d'entreprendre, et sa surtout réussite, D fait le choix de se raconter comme un « héros » : ce choix apparaît décalé par rapport aux autres interviewés qui expliquent leurs raisons d'agir avec davantage de modestie, tandis que $\mathrm{D}$ semble chercher à susciter l'admiration. Le fait que selon les cas, les EC de notre corpus aient été interviewés par un ou une étudiant(e), ou par un ou deux étudiant(e)s, tout comme l'écart d'âge entre les intervieweur(euse)s et les interviewé(e)s peut expliquer ce décalage. En effet, $\mathrm{D}$ est le seul à être interviewé par deux étudiantes. On ne peut en effet interpréter un récit sans tenir compte de la situation de recueil qui agit nécessairement sur les façons de les dire et plus globalement les façons de (se) raconter. (cf. caractéristiques de passation des entretiens en annexe 2).

\section{Approche herméneutique de Ricœur: insistance sur une temporalité explicative rétrospective}

L'approche de Ricœur $(1983,1984,1985)^{29}$, - qui a développé les notions (liées) de " configuration » et « d'identité narrative » (1990) -, a insisté sur l'aspect " rétrospectif » de la temporalité narrative ; cette dimension rétrospective apparait liée aux nombreuses explications, justifications et interprétations permettant d'expliquer les conduites passées des narrateurs (et/ou de leurs personnages).

\section{La notion de mise en intrigue (mimesis II) : dimensions épisodique et « configurante»}

Ricœur a souligné que la temporalité racontée (mimsésis II) est en interaction avec la temporalité du vécu (mimésis I) et celle de la lecture (mimésis III). En l'intégrant à la notion de mimesis $I^{30}$, Ricœur a caractérisé le récit dans son rapport au temps, en soulignant deux dimensions : épisodique et configurante. La dimension épisodique du récit se définit comme tirant le temps narratif du côté de la représentation linéaire: les épisodes se suivent l'un l'autre en accord avec l'ordre irréversible du temps commun aux événements physiques et humains. La dimension configurante, en retour, présente les traits temporels inverses de ceux de la dimension épisodique et renvoie à l'idée que les événements successifs doivent pouvoir être « saisis ensemble » de façon rétrospective :

l'arrangement configurant transforme la succession des événements en une totalité signifiante qui est le corrélat d'assembler les événements et fait que l'histoire se laisse suivre [....]. En lisant la fin dans le commencement et le commencement dans la fin, nous apprenons à lire le temps lui-même à rebours, comme la récapitulation des conditions initiales d'un cours d'action dans ses conséquences terminales. (1983: 130)

Le rôle joué par les nombreuses explications, argumentations, justifications, interprétations dans cette lecture à rebours est fondamental : ce sont ces dernières qui permettent de comprendre rétrospectivement l'enchaînement des événements et ou des actions des personnages; dit autrement Ricœur a insisté sur le fait qu'une narration 
permet de "configurer» la temporalité, en mettant l'accent sur la dimension " rétrospective» de cette configuration. Précisons que cette lecture rétrospective des événements n'empêche pas que soient mentionnés des projets. Ricœur a ainsi souligné que le récit était toujours inclus dans une dialectique de remémoration et d'anticipation, et mentionné (de manière allusive) que cela le teinte d'une temporalité affective, à savoir celle du souci (du futur) :

parmi les faits racontés à un temps du passé, prennent place des projets, des attentes, des anticipations, par quoi les protagonistes sont orientés vers leur avenir mortel [...]. Autrement dit, le récit raconte aussi le souci. [...]. C'est pourquoi il n'y a pas d'absurdité à parler de l'unité narrative d'une vie, sous le signe de récits qui enseignent à articuler narrativement rétrospection et prospection. (1990: 192-93)

\section{La notion d'identité narrative : conciliation de la mêmeté (noyau constant du soi) et de l'ipséité (différences de soi à soi) dans et par l'activité narrative}

46 A partir de la notion de mise en intrigue, Ricœur a élaboré la notion "d'identité narrative » (1990). Il l'a présentée comme permettant d'éliminer le paradoxe suivant: nous avons tous le sentiment de nous transformer au cours de notre existence, tout en ayant simultanément le sentiment d'une continuité (ou permanence) de notre soi. Ce paradoxe est temporel et plus globalement existentiel.

L'identité narrative permet de concilier, par l'activité narrative, deux aspects d'un même processus :

48 - la mêmeté (idem) comme constance du soi ou «maintien de soi » à travers les diverses étapes de la vie ; la mêmeté renvoie à un noyau permanent et identifiable du soi malgré le temps qui passe, à une sorte de continuité ou d'invariant du soi (certains aspects du « caractère » par exemple);

- et l'ipséité (ipse) qui renvoie au contraire aux aspects changeants et mouvants du soi, aspects que l'on ne retrouve justement pas dans le «noyau permanent et identifiable " mentionné ci-dessus.

49 Ce processus est toujours inachevé : chaque récit peut être le prétexte à une nouvelle mise en intrigue, un nouveau regard rétrospectif expliquant l'enchaînement des événements ou les raisons d'agir, une nouvelle configuration discursive entre "ipséité " et « mêmeté ».

50 Ainsi, Ricœur ne conçoit pas la notion d'identité comme un état ou une essence de la personne mais comme un processus visant à assurer une régulation dans la présentation d'un soi changeant en permanence.

51 En outre, Ricœur insiste sur le point suivant dans son approche de l'identité narrative :

l'interprétation de soi intègre deux grands types de récit, historique et de fiction :

$\mathrm{Ne}$ tenons-nous pas les vies humaines pour plus lisibles lorsqu'elles sont interprétées en fonction des histoires que les gens racontent à leur sujet? Et ces histoires de vie ne sont-elles pas rendues à leur tour plus intelligibles lorsque leur sont appliqués des modèles narratifs - des intrigues - empruntées à l'histoire proprement dite ou à la fiction (drame ou roman) ? Il semblait donc plausible de tenir pour valable la chaîne suivante d'assertions : la compréhension de soi est une interprétation; l'interprétation de soi, à son tour, trouve dans le récit, parmi d'autres signes et symboles, une médiation privilégiée ; cette dernière emprunte à l'histoire autant qu'à la fiction, faisant de l'histoire de vie une histoire fictive, ou, si 
l'on préfère, une fiction historique, entrecroisant le style historiographique des

biographies au style romanesque des autobiographies imaginaires. (1990:138)

Ainsi l'identité narrative convoque nécessairement une part de récit fictionnel et donc d'invention: un récit n'est pas un pur décalque de la réalité car l'activité narratrice introduit une part de créativité et permet de travailler cette identité.

\section{Des processus identitaires qui se racontent et se renouvellent à chaque récit}

53 On a donc toujours affaire à des " processus identitaires » qui ne sont ni fixes, ni figés et peuvent se dire de façon renouvelée, cela en trouvant rétrospectivement de nouveaux liens entre les événements et en les adaptant à la situation. La notion d'identité narrative au sens de Ricœur croise en partie les propositions de sociologues comme Sainsaulieu (1977), Dubar (1991), ou encore de l'historien Pollack (1993) qui ont, chacun à leur manière, proposé de sortir d'une vision stable de l'identité.

Il est ainsi aujourd'hui admis que dans la façon de "configurer» ou de "mettre en intrigue» les événements, - c'est-à-dire de les expliquer, justifier, interpréter rétrospectivement-, le narrateur peut (ré)inventer et (re)construire son identité (narrative) à chaque nouvelle "version » de son récit biographique ; cela en «trouvant » rétrospectivement de nouveaux liens entre les événements ou de nouvelles raisons d'agir, et en donnant ce faisant une cohérence à un ensemble d'événements qui apparaissaient jusque-là sans lien entre eux. C'est cette dimension que Bourdieu a qualifié « d'illusion biographique ", dans la mesure où, selon lui, des liens de causalité seraient finalement créés dans et par l'acte de (se) raconter pour la seule nécessité formelle du récit; c'est aussi ce qui fait le succès actuel des "méthodes narratives" dans le domaine du développement personnel.

Cela dit, dans une conception du langage non représentationniste mais « située » (Boutet 1989, Vion 1992, Mondada 2001), c'est-à-dire une conception où le langage ne reflète pas l'expérience mais la reconstruit, cette dimension d'illusion ou d'invention n'est pas à attribuer à l'acte de narration en lui-même mais à tout type de discours : toute situation de langage peut être vue comme une pratique sociale permettant aux locuteurs de «travailler» les significations sociales et donc de les (co)produire. Cette conception s'inscrit plus globalement selon nous dans une perspective dialogique (Bakhtine 1970) selon laquelle (i) l'activité langagière est toujours « située » dans un contexte social donné et (ii) tout locuteur dit ce qu'il a à dire d'abord et avant tout en fonction de l'interlocuteur à qui il s'adresse, et plus globalement en fonction de la façon dont il interprète la situation dans laquelle il se trouve.

De ce point de vue, la «version » que proposent les interviewés de notre corpus pour raconter leur parcours est à mettre en étroite relation avec le fait suivant: ils ont interprété la situation comme une demande (de la part des intervieweurs) d'aide et de conseils pour s'insérer prochainement sur le marché de travail, et dans une forme de compréhension responsive (dialogisme responsif), ils ont anticipé les réactions de leurs interviewers en évitant de les décourager ou de les inquiéter : c'est ce qui expliquerait pourquoi ils se racontent sans s'attarder sur les aspects négatifs de leur parcours ou de leur activité actuelle. L'un d'entre eux indique d'ailleurs clairement cette position sur un mode humoristique : 


\begin{tabular}{|l|l|l|}
\hline A14 & 16 & moi c'est un métier que je recommande / \\
\hline A14 & 17 & mais bon maintenant c'est trop tard vous êtes foutus / <rires> \\
\hline A14 & 18 & vous êtes là euh je n'aurai plus le temps de vous décourager / \\
\hline
\end{tabular}

Le fait de taire les aspects négatifs du parcours donne parfois lieu à des enchainements quelque peu abrupts :

\begin{tabular}{|l|l|l|}
\hline Y1 & 1 & pouvez-vous me raconter en quelques minutes votre parcours ? \\
\hline B1 & 1 & j'ai commencé par de l'auDIT / chez $\mathrm{C}^{31} /$ \\
\hline B1 & 2 & je me suis fait virer / $(1 \mathrm{sec})$ \\
\hline B1 & 3 & $\begin{array}{l}\text { parce que: je sais plus pourquoi euh:::/ <ton désabusé> <j'avais pas l'esprit C / xxxx tout } \\
\text { simplement je crois> / }\end{array}$ \\
\hline B1 & 3 & $\begin{array}{l}\text { parce que: je sais plus pourquoi euh:::/ <ton désabusé> <j'avais pas l'esprit C / xxxx tout } \\
\text { simplement je crois> / }\end{array}$ \\
\hline Y2 & & est-ce que vous pourriez essayer de ramener fin :::je sais pas le micro xxxx / \\
\hline B2 & 2 & $\begin{array}{l}\text { bon euh:::ensuite j'ai été euh:: j'ai fait une carrière en Allemagne pendant trois ans / } \\
\text { dans trois boîtes différentes }\end{array}$ \\
\hline B2 & 1 & ah ouais d'accord voilà- \\
\hline & & \\
\hline
\end{tabular}

On n'apprendra que plus tard (B49) dans l'entretien ce qu'est «l'esprit C» selon B (à savoir : être centré sur la rentabilité, cela en ayant une activité répétitive et spécialisée et en négligeant toute forme de convivialité avec les clients); on apprendra également en quoi cet esprit (d'un grand cabinet) s'oppose en tout point à celui d'un petit cabinet, ce qui aboutira à valoriser fortement les valeurs et l'activité du travail en petit cabinet et à dévaloriser celles des «grands» cabinets internationaux (nommés «Big 4 » par les professionnels : PWC, KPMG, Deloitte, Ernst \& Young) ${ }^{32}$.

\section{Des explications et des arguments récurrents pour expliquer le choix de travailler en petit cabinet}

Le point de vue de B est représentatif de l'ensemble des interviewés travaillant en petit cabinet: dans la narration de leur parcours, les interviewés avancent tous les mêmes « raisons " pour justifier rétrospectivement leur choix de travailler dans une petite structure, cela en employant souvent les mêmes expressions et les mêmes façons de dire : 
tous expliquent que travailler en petit cabinet est (i) moins stressant du fait de l'absence du poids de la hiérarchie ; (ii) plus intéressant car l'activité y est plus diversifiée (on peut « s'occuper d'un dossier de $\mathrm{A}$ à $\mathrm{Z}$ »), (iii) plus stimulant car le statut d'indépendant permet d'être plus autonome et de prendre plus de risques ; (iv) plus convivial car le " contact ", la «proximité », le « relationnel » avec le client sont plus forts. Ces aspects sont décrits comme compensant les horaires plus lourds et une rémunération moindre en petit cabinet.

Cette comparaison aboutit à critiquer en creux le mode de travail et l'idéologie des grands cabinets actuellement en train de s'imposer sur le marché du travail (au niveau international comme national). Néanmoins, tous les interviewés insistent sur l'aspect formateur et la légitimité qu'apporte une expérience dans l'un des Big Four sur le marché du travail, en utilisant souvent les mêmes expressions. Plusieurs interviewés emploient ainsi la métaphore du «tremplin » ou de la « carte de visite » (entretiens $1,6,9,11$ ) pour conseiller une telle expérience :

\begin{tabular}{|l|l|l|}
\hline A14 & 42 & $\begin{array}{l}\text { et donc euh et puis ça fait une belle carte de visite } \\
/\end{array}$ \\
\hline A14 & 43 & et ça apprend plein de choses / \\
\hline
\end{tabular}

61 Les critiques (des grands cabinets » sont faites en creux ou atténuées, mais aussi nuancées puisque les points positifs sont aussi mentionnés. Dit autrement, les interviewés semblent contrôler leurs propos à l'égard des grands cabinets, ce que l'on tentera d'expliquer en conclusion en reliant ces entretiens biographiques à leur contexte de production local (conditions d'énonciation) comme global (contexte socio-professionnel et idéologique).

62 Comme on va le voir dans le point suivant, une autre caractéristique va apparaître commune aux différents récits de notre corpus, à savoir la mise en mots d'une temporalité affective paradoxale dont le côté négatif va apparaitre là aussi atténué.

\section{Approches interactives du récit : émergence d'une dimension affective}

63 Les premières approches interactives du récit ont été inspirées principalement par les recherches du sociolinguiste Labov $(1978,1997,2007)$. Elles se sont redéveloppées ensuite dans des perspectives analysant le fonctionnement conversationnel et/ou dialogique ${ }^{33} \mathrm{du}$ récit dans l'entretien (entre autres: François 2004 [1988], Bres 1994, Laforest 1996, Carcassonne et al. 2001, Nossik 2011).

En s'intéressant à des récits oraux et non plus écrits, ces perspectives ont remis en cause les approches structuralistes ou de Ricœur en n'appréhendant plus le récit comme un résultat fini et coupé du social mais en tant qu'activité de co-construction narrative, étroitement liée au contexte (local comme global) de recueil. Les questions d'organisation temporelle ou de structure sont passées au second plan pour s'interroger davantage sur les questions suivantes: qu'est-ce qui est induit par l'un ou l'autre des interlocuteurs pour co-construire le récit, qu'est-ce qui est induit par la situation de recueil elle-même ? Que permet de découvrir l'analyse de l'enchainement des énoncés entre les prises de 
parole ou au sein d'une même prise de parole? Ces approches ont par exemple montré comment, dans un récit recueilli en interaction, l'interlocuteur peut questionner les trous de la narration, pointer ce qui l'étonne, etc. : et ce faisant, réorienter le cours de récit et participer à sa construction.

Ces approches ont de plus souligné le véritable travail langagier occasionné par la pratique de l'entretien qui permet des (co)productions de sens parfois inattendues pour les interlocuteurs eux-mêmes, lesquels n'apparaissent pas spécifiquement soucieux de la mise en cohérence des événements racontés. Plus globalement, ces perspectives ont montré qu'il n'existait pas un seul type de récit mais une famille de conduites narratives, lesquelles se diversifient en fonction de ce qui est à raconter, de l'interlocuteur à qui on s'adresse, de récepteurs absents dont les attentes sont projetées par les interviewés comme par les intervieweurs, du lieu et du temps dont on dispose, etc.

\section{Mise en évidence d'un fonctionnement spécifique de la narration dans l'interaction}

\section{Spécificités du récit conversationnel}

Les analyses conversationnelles américaines se sont surtout attachées à analyser le fonctionnement de l'enchaînement des énoncés dans l'interaction, tour de parole après tour de parole. Elles ont amené certains chercheurs à examiner la façon dont un récit se développe dans une interaction qu'il s'agisse de la façon dont il est introduit dans le cours de l'échange, dont il se poursuit ou dont il se termine (cf. Sacks 1995, Ochs et Caps 2001 pour des travaux anglo-saxons; Bres 1995, Revaz et Filliettaz 2006, pour des travaux en langue française). Ces recherches ont en outre insisté sur le fait que différents types de discours peuvent se repérer dans une interaction et se doter alors de certaines des caractéristiques de l'interaction en question: l'expression "récit conversationnel » renvoie dans ce type d'approche à l'insertion, voire à la fusion du type de discours "récit» dans le type d'interaction "conversation $»^{34}$. Un "récit conversationnel» présente souvent l'une des fonctions de la "conversation", à savoir celle du maintien d'un lien social. De ce fait, ce type de récit ne correspond pas toujours à des récits « fortement racontables » comme ceux analysés par Labov, mais à des récits à faible degré de racontabilité (au sens de Ochs et Capps 2001, infra) dont la fonction est plus phatique ou ludique (Coates 1997, 2001) qu'intrigante ou configurante (au sens de Baroni, cf. supra).

Ochs et Capps (2001) ont par exemple pris pour objet les récits rendant compte des "événements de la vie» («life events») dans la conversation ordinaire, les "living narrative ». Leur analyse montre que ces derniers sont "modelés et remodelés tour de parole par tour de parole dans le cours de la conversation » $(2001: 2)^{35}$, et qu'on ne peut de ce fait dégager de façon simple une «structure » concernant ces récits. Les auteurs proposent alors d'étaler la diversité des récits qui peuvent émerger des conversations sur un continuum allant des "récits par défaut " (default narrative) aux "récits ouverts » (open narrative). Elles mettent en évidence cinq dimensions permettant de contraster les deux pôles de ce continuum: tellership (nombre de narrateurs actifs), tellability (racontabilité), embedeness (enchâssement), linearity (linéarité), moral stance (posture morale); ce que l'on peut synthétiser dans le tableau comparatif suivant : 


\begin{tabular}{|l|l|l|}
\hline & $\begin{array}{l}\text { récits "par } \\
\text { défaut " } \\
\text { (Ochs et Capp, } \\
2001: 20)\end{array}$ & $\begin{array}{l}\text { "ouverts " } \\
\text { (Ochs et Capp, } \\
2001: 23)\end{array}$ \\
\hline $\begin{array}{l}\text { nombre de narrateurs actifs } \\
\text { (tellership) }\end{array}$ & un seul & plusieurs \\
\hline degré de « racontabilité » (tellability) & forte & faible \\
\hline $\begin{array}{l}\text { degré d'enchâssement dans la conversation et dans } \\
\text { l'activité environnante (embedeness) }\end{array}$ & $\begin{array}{l}\text { faible } \\
\text { (détachement fort) }\end{array}$ & fort \\
\hline $\begin{array}{l}\text { degré de linéarité ou d'organisation temporelle et } \\
\text { causale (linearity) }\end{array}$ & forte & faible \\
\hline posture morale (moral stance) & $\begin{array}{l}\text { constante } \\
\text { certaine }\end{array}$ & $\begin{array}{l}\text { fluide } \\
\text { incertaine }\end{array}$ \\
\hline
\end{tabular}

On peut ainsi trouver quantité de formes intermédiaires sur ce continuum (allant du récit "par défaut» au récit «ouvert») et sur lequel les cinq aspects bougent plus ou moins ensemble.

69 La perspective d'Occhs et Capps permet donc de prendre une distance avec les approches du récit en termes de "structure ", et de ne plus considérer le critère de l'organisation temporelle et/ou causale comme central, contrairement aux approches structuralistes ou à celle de Ricœur. Ochs (2004) précise ainsi que deux «tendances » peuvent être dégagées concernant la pratique de récits d'expérience personnelle : d'une part celle qui permet de trouver une logique d'événement cohérente, d'autre part celle qui permet, dans un cadre dialogal, de mettre à l'épreuve des logiques alternatives. Ces deux tendances impliquent des «aptitudes narratives » différentes: «présenter une logique d'événements sûre et cohérente » vs « questionner, mettre à l'épreuve et réviser une logique d'événements ».

\section{Spécificités du récit dans l'entretien}

70 A côté des spécificités du récit conversationnel, celles du récit dans l'entretien ont également été envisagées dans une perspective dialogique ${ }^{36}$, ce qui a permis d'analyser la temporalité sous un autre angle que dans les approches inspirées des structuralistes ou de Ricœur.

71 Nous avons montré par exemple (Carcassonne 2006, 2007) que dans un entretien, deux niveaux de récits pouvaient être distingués :

72 - le premier niveau correspond aux récits ponctuels produits en réponse à certaines demandes de récits ;

- le deuxième niveau correspond au récit global que le récepteur reconstitue (i) à partir de la somme des récits et arguments produits ponctuellement au cours de l'entretien, mais aussi (ii) à partir des informations qui se dessinent en creux entre les différentes périodes évoquées au cours de l'entretien: pour reconstituer cette sorte de "récit des récits » 
(Kaufmann, 2004 : 157), c'est parfois le récepteur qui réordonne certains événements et/ ou suppose ce qui n'a pas été explicité, sans que l'on puisse savoir si l'interviewé fait ces mêmes liens. Notons que ce rôle de l'interprète-récepteur n'est pas propre à la seule situation d'entretien : il est tout aussi net pour les films (qui sont découpés et montés en séquences), pour les bandes dessinées (où il se passe quelque chose entre les images), et concerne finalement plus ou moins toute forme de narration.

Dans le corpus ici présenté, l'analyse a montré que la temporalité ne se caractérisait pas avant tout par le fait d'être « organisée » par les (co)narrateurs (que cela soit sur le mode du suspense comme dans les approches structuralistes ou sur le mode de l'explication rétrospective comme dans les approches herméneutiques inspirées de Ricœur) mais par une dimension affective paradoxale: au-delà de leurs différences de "style ${ }^{37}$, les différents interviewés insistent tous (i) sur l'indépendance et la liberté d'entreprendre comme deux valeurs guidant leur vie professionnelle, (ii) tout en soulignant le manque de temps par rapport à la masse de travail à effectuer, manque de temps les obligeant à travailler en permanence (tard le soir et week-end compris) et à mener une vie finalement très dépendante de cette forte contrainte temporelle.

Ce paradoxe n'est jamais souligné explicitement (rappelons qu'il ne s'agit pas d'une temporalité que le narrateur organise mais d'une temporalité qui l'affecte) : c'est en mobilisant une perspective interprétative dialogique insistant ici sur les mouvements discursifs autodialogiques (cf. point 1.1), c'est-à-dire en rapprochant des contenus évoqués à distance les uns des autres que l'on voit se dessiner cette temporalité qui n'émerge donc pas de la lecture du premier récit ponctuel du parcours de l'interviewé, mais suite aux questions ultérieures, demandant par exemple de raconter une journée de travail ou demandant quels sont les inconvénients du métier. Les réponses aboutissent alors à réinterpréter le premier récit initial en le teintant de cette temporalité affective caractérisée par une soumission librement consentie à une activité de travail chronophage dominant tous les autres aspects de la vie de l'interviewé. Les interviewés présentent tous cet aspect chronophage de leur activité en essayant d'atténuer sa portée, notamment en insistant sur les avantages du métier (en particulier la rémunération), tenant certainement compte en cela, on l'a vu, du fait que leurs intervieweurs s'apprêtent à suivre la même voie qu'eux.

L'indépendance apparait finalement très relative dans le discours des interviewés puisqu'ils se présentent comme étant obligés des se consacrer totalement à leur activité de travail, et cela alors même qu'ils ont choisi de travailler en petit cabinet pour être plus indépendant.

\section{Commentaire d'un deuxième extrait de l'entretien de D (annexe 5)}

$\mathrm{D}$, on l'a vu, «fanfaronne » dans un premier récit ponctuel où domine la temporalité affective de la fierté (cf. extrait déjà commenté et joint en annexe 4), en mettant en avant son indépendance, sa liberté d'entreprendre, sa réussite.

La lecture de l'extrait joint en annexe 5 reconfigure ensuite fortement l'interprétation de ce premier extrait : la vie de D y est présentée de façon moins idéale : un certain regret ou manque se dit en creux quand est évoqué (1.3) le fait de « sacrifier " ${ }^{38}$ la vie familiale ${ }^{39}$ :

La mise en rapport des deux extraits joints en annexe 4 et 5 nous présente ainsi une nouvelle image de D: s'il est certes en accord avec ses valeurs (i) de liberté 
d'entreprendre, (ii) -et bien qu'il s'en défende- de gain d'argent, (iii) il regrette de ne pas avoir de vie de famille.

Pour exprimer ce regret, on note que D s'engage beaucoup moins dans son discours que dans le premier extrait, à nouveau certainement pour ne pas inquiéter ses intervieweuses. Il évite ce faisant d'insister sur le fait que l'activité d'EC nécessite de consacrer presque tout son temps à son activité de travail, et se débrouille pour le dire en creux: les marques de "subjectivité énonciative» (Kerbrat-Orecchioni 1980) sont quasiment absentes, D tenant un discours générique (« on », verbes à l'infinitif, absence de possessif : «la » vie et non «ma » vie). Il généralise ce qu'il dit à propos de son activité à « tout emploi qui nécessite une passion » (1.1), à « toutes les professions libérales » (1.7).

Dans cet extrait, on note un premier mouvement argumentatif (l. 1 à 9) qui est reprismodifié aux lignes 10 à 21 puis aux 22 à 25 (autodialogisme). Dans ce triple mouvement argumentatif, D insiste plusieurs fois sur les mêmes aspects :

81 Après avoir précisé à la fin du premier mouvement qu'il n'y avait pas de «solution miracle » (1.9) par rapport à cet inconvénient, il redit néanmoins comme il l'avait déjà dit (1.4) que «c'est quelque chose qui peut être géré» (l.11), puis il liste les avantages du métier (1. 12 à 20) : voyages, contacts, découvertes, rémunération (il indique même son salaire).

Ce mouvement argumentatif est repris-modifié aux lignes 22 à $25: \mathrm{D}$ revient ainsi spontanément (1.22) à « cet aspect de la vie familiale qui est un peu sacrifié » et reproduit le même mouvement argumentatif, en généralisant à nouveau cet inconvénient à d'autres professions (1.23), pour rappeler ensuite que cela se gère (1. 24 « simplement il faut être organisé $/$ ) et finalement indiquer (de façon atténuée) qu'il s'agit néanmoins d'un inconvénient : « 1.25 c'est cet aspect qui est un peu::: gênant »).

On note dans ce triple mouvement argumentatif plusieurs atténuations : le terme "un peu $(1.6,8,22,25)$ est ainsi utilisé à quatre reprises pour atténuer la portée du regret d'une vie familiale sacrifiée.

\section{Autres extraits}

Les autres interviewés travaillant en petit cabinet mettent eux aussi en mots cette temporalité affective paradoxale: tous les interviewés mettent en avant tantôt les avantages de «l'indépendance ", tantôt les inconvénients de la contrainte horaire. Nous présentons en annexe 6, 7, 8 et 9 des extraits représentatifs de ce contraste.

Ces extraits entrent clairement en écho avec les extraits de D que nous avons plus précisément analysés ci-dessus : dans le premier extrait joint en annexe 6, A insiste sur l'importance de l'indépendance en petit cabinet, comme il le fait à plusieurs reprises au cours de l'entretien. Ultérieurement, et comme le montre l'extrait représentatif joint en annexe 7, A soulignera à plusieurs reprises la lourdeur des horaires, tout en atténuant, comme $\mathrm{D}$, cet inconvénient.

De façon proche (annexe 8), B met en rapport l'indépendance avec la lourdeur des horaires tandis que C (annexe 9) mentionne les avantages de l'indépendance et de la variété tout en insistant sur le fait qu'elles ont un " prix ».

Ces contrastes renvoyant à une temporalité affective paradoxale se retrouvent plusieurs fois au sein de chaque entretien. Comme on l'a vu, cette temporalité ne se dégage pas en analysant la « structure » narrative chronologique, mais en mettant en rapport différents 
extraits de l'entretien, les extraits ultérieurs reconfigurant le sens des extraits antérieurs (analyse de l'autodialogisme).

\section{Conclusion} effet sur le récepteur.

Nous avons vu qu'historiquement, le récit a d'abord été défini à partir de critères internes (en particulier en termes de structure) pour s'ouvrir ensuite à des définitions (i) prenant de plus en plus en compte les situations dans lesquelles il est produit mais aussi reçu, (ii) remettant progressivement en cause l'idée même d'une structure du récit.

Nous avons ainsi présenté trois "approches » théoriques du récit en les reliant à trois dimensions de la temporalité racontée, et en insistant sur la diversité des formes narratives: le "récit» au sens structuraliste ne correspond qu'à une seule sorte de « récit », d'autres formes de récit « en interaction » étant attestées, lesquelles ne mettent pas au centre de leur analyse la temporalité chronologique ou configurationnelle.

L'approche structuraliste considère le récit comme fermé sur lui-même et accentue une temporalité prospective et en tension vécue par un « héros » surmontant des épreuves. Cette approche structuraliste n'a été éclairante que pour un seul récit de notre corpus, cette présentation de soi n'ayant pas été privilégiée par les interviewés.

1 L'approche herméneutique de Ricœur considère au contraire le récit comme une configuration des événements (ré)interprétés par un regard rétrospectif. Cette approche nous a amenée à pointer la récurrence des mêmes raisons et arguments (et des mêmes façons de les dire) pour expliquer le choix de travailler en petit cabinet et critiquer ce faisant en creux le mode de travail et l'idéologie des grands cabinets, mais aussi à pointer la récurrence des mêmes expressions pour valoriser certains aspects de l'expérience en grand cabinet. Les interviewés conseillent tous une expérience dans l'un des grands cabinets tout en critiquant en creux le mode de travail et l'idéologie de ces derniers, c'està-dire en nuançant voire en contrôlant leurs critiques des grands cabinets.

L'approche dialogique dans laquelle nous nous inscrivons a permis quant à elle de dégager des temporalités narratives affectives, lesquelles se dessinent dans la dynamique du discours. Comme on l'a vu en comparant précisément deux extraits de l'entretien de D, il s'agit dans ce type d'approche de mettre en rapport certains extraits avec d'autres (autodialogisme) pour interpréter le sens émergeant en creux de cette mise en rapport; ils s'agit aussi d'analyser les extraits dans leur déroulement linéaire, en accordant autant d'importance au contenu, à la façon de dire ce contenu, à sa relation au contexte, et à son

Certaines temporalités affectives sont dans cette perspective apparues spécifiques à certains interviewés, par exemple celle de la fierté chez D; tandis qu'une autre est apparue commune à tous les interviewés, à savoir une temporalité affective mettant en tension un sentiment d'indépendance d'une part, de fortes contraintes horaires d'autres part (ces dernières débouchant sur le regret d'une vie familiale).

4 Ainsi, tous les interviewés du corpus ayant quitté les grands cabinets pour des plus petits mettent en mots une temporalité affective caractérisée par une forme de soumission librement consentie à une activité de travail chronophage dominant tous les autres aspects de la vie. En même temps et malgré tout, cette temporalité est présentée en creux comme beaucoup plus agréable à vivre que celle des grands cabinets, en particulier parce 
que le contact avec le client est plus chaleureux, l'activité plus diversifiée, la hiérarchie moins pesante.

Il y a ainsi des aspects récurrents repérés dans la pratique narrative des experts comptables du corpus ici analysé : mêmes arguments pour justifier le choix de travailler en petit cabinet et critiquer en creux le travail en grand cabinet, mêmes expressions pour valoriser malgré tout certains aspects de l'expérience en grand cabinet, mêmes nuances et contrôle des propos vis-à-vis des critiques concernant les grands cabinets, même temporalité affective paradoxale décrivant l'expérience en petit cabinet, même absence d'explicitation de ce paradoxe.

En mettant en relation les aspects récurrents de ces récits avec les conditions de production locales (situation de recueil) et globales (contexte socio-professionnel et/ou idéologique), on peut émettre pour conclure un certain nombre d'hypothèses (qui demanderaient à confirmées sur un corpus plus important) pour expliquer ces aspects récurrents :

97 - au niveau du contexte local de recueil des entretiens biographiques, les interviewés tiendraient compte de leurs interlocuteurs s'apprêtant à entrer sur le marché de travail (dialogisme responsif) en évitant (pour ne pas les inquiéter) de critiquer trop ouvertement les grands cabinets dans leurs récits,

- au niveau du contexte socio-professionnel, une forme de «domination» des grands cabinets sur les petits » (cf. note 32) agirait sur le marché du travail comme sur la scène de l'entretien. Les EC travaillant en petits cabinets seraient fortement soumis à l'idéologie et à l'imposition des normes des grands cabinets, ce qui les empêcherait de critiquer trop ouvertement ces derniers. Les récits seraient ainsi traversés par ces rapports de force qui expliqueraient la récurrence de certains contenus et de certaines façons de les dire. Ces rapports de force structureraient finalement ces récits dans un certain «registre discursif » (Achard 1993) ${ }^{40}$, voire une « formation langagière » (Boutet et al. 1976) ${ }^{41}$.

- Plus globalement encore, on peut se demander si l'absence d'explication de la temporalité affective paradoxale décrivant l'expérience en petit cabinet ne pourrait pas être mise en rapport avec l'idéologie néo-libérale telle que l'a caractérisée Bihr (2006). Ce dernier a en effet insisté sur la tension qui traverse cette idéologie, tension qui pèserait directement sur la vie professionnelle comme personnelle des individus. Cette tension serait due à l'impératif constamment présent d'un «devoir d'entreprendre » (par une action autonome et libre), impératif qui ne serait possible que par une forme de soumission librement consentie à un certain nombre de règles capitalistes. L'individu concerné par ces idéologies ne pourrait se vivre - et se dire - libre d'entreprendre et autonome qu'en acceptant un certain nombre de règles, dans une forme de double contrainte qui serait tout à la fois difficile à vivre et à dire.

\begin{tabular}{|l|l|l|}
\hline C11 & 2 & écoutez /l'avantage majeur pour moi / mais ça a un prix:: / c'est l'indépendance / mmh \\
\hline C11 & 3 & / je vous l'ai dit / c'est pour ça que j'ai choisi ça / \\
\hline C11 & 4 & euh:::vous êtes votre propre décideu:r / \\
\hline C11 & 5 & donc pour moi c'est vraiment tRES important / \\
\hline
\end{tabular}




\begin{tabular}{|l|l|l|l|}
\hline C11 & 6 & $\begin{array}{l}\text { euh::: y a pas que ça / les avantages aussi c'est que c'est une activité extrêmement } \\
\text { VAriée / }\end{array}$ & \\
\hline C11 & 7 & euh parce que la clientèle est variée / & \\
\hline C11 & 8 & en tout cas on l'espère / en tout cas chez nous elle variée / & \\
\hline C11 & 9 & euh parce que chaque dossier est un cas différent / & \\
\hline
\end{tabular}

\section{BIBLIOGRAPHY}

Achard P., 1993, Sociologie du langage, Paris, PUF.

Adam J.M., 1992, Les textes : types et prototypes, Paris, Armand Colin.

Augustyn, M., Grossmann, F., 2009, « Je nage dans la joie, la colère me submerge - Etude de quelques métaphores spatiales dans le champ des affects » dans Marie J. Berchoud (éd.), Les mots de l'espace, entre expression et appropriation, pp. 57-76, Paris, L'Harmattan.

Authier Revuz J., 1982, « Hétérogénéité montrée et hétérogénéité constitutive : éléments pour une approche de l'autre dans le discours ", dans D.R.L.A.V. 26, p. 91-151.

- 1995, Ces mots qui ne vont pas de soi, Paris : Larousse (2 vol).

Bakhtine M. 1970 [1963] [1929], La Poétique de Dostoïevski, trad. du russe par Isabelle Kolitcheff, Paris, Seuil, coll. « Pierres vives ».

- 1977 [1929], voir Volochinov.

- 1978 [1975/1934], « Du discours romanesque », dans M. Bakhtine, Esthétique et théorie du roman, Paris, Gallimard, p. 83-233.

- 1984 [1979]), Esthétique de la création verbale, Paris, Gallimard.

Baroni R., 2009, L'CEuvre du temps, Paris, Seuil.

Barthes R., 1966, «Introduction à l'analyse structurale des récits », dans Communications n8, p. 1-27.

Bertaux D., 1997, Les récits de vie, Paris, Nathan.

Bihr A., 2006, «L'idéologie néolibérale », dans Semen [En ligne], 30 | 2011, mis en ligne le 01 janvier 2011, consulté le 06 mars 2013. URL : http://semen.revues.org/8960.

Bourdieu P., 1986, «L'illusion biographique », dans Actes de la Recherche en Sciences sociales 62-63, p. 69-72.

Boutet J., 1989, Construire le sens, Neuchâtel (Suisse), Peter Lang.

Boutet J., Fiala P., Simonin-Grumbach J., 1976, « Sociolinguistique ou sociologie du langage ? ", Critique $\mathrm{n}^{\circ} 344$, p. 68-85.

Bres J., 1994, La Narrativité, Duculot.

- 1995, « “Alors raconte !”. La négociation du récit dans l'interaction de l'interview », dans D. 
Véronique et R. Vion (éds). Des savoirs-faire communicationnels, Aix-en-Provence, Publication de l'université de Provence, p. 289-301.

- 2005, Savoir de quoi on parle : dialogue, dialogal, dialogique ; dialogisme, polyphonie. In J. Bres, P. Haillet, S. Mellet, H. Nølke, \& L. Rosier (Eds.), Dialogisme et polyphonie. Approches linguistiques. Bruxelles :De Boek Duculot, p. 47-61.

Bronckart J. P., Bota C., 2011, Bakhtine démasqué, Histoire d'un menteur, d'une escroquerie et d'un délire collectif, Genève, Droz.

- 2014, «Bakhtine démasqué », COnTEXTES [En ligne], Prises de position, mis en ligne le 24 juin 2014. URL : http://contextes.revues.org/5971

Carcassonne M., 2004, « De la dimension temporelle des affects vécus à celle des affects racontés : quelles médiations? ", dans Delamotte-Legrand G. (dir.), Les médiations langagières, vol. 1, Des faits de langue aux discours, Actes du colloque international La médiation: marquages en langue et en discours, Dyalang CNRS : Publications de l'Université de Rouen, pp. 403-411. http://basepub.dauphine.fr/ handle/123456789/1234

- 2006, « Présentation de soi dans l'entretien : narration, explication, interprétation », dans Grossen M., Salazar-Orvig A. (dirs.), L'entretien clinique en question : analyse des d'un genre communicatif hétérogène, Paris, Belin (coll. Belin Sup), chap. 10, pp. 213-231.

- 2007, "Sens, temps, et affects dans des récits de vie recueillis en interaction ", dans Vox Poetica, Passion et narration, Revue en lignes, http://www.vox-poetica.org/t/pas/carcassonne.htm, pp.1-29.

- 2010, « Les voix dans un entretien de recherche : quelles marques et quelles fonctions interactionnelles et sociales? ", dans Neveu F., Muni Toke V., Klinger T., Durand J., Mondada L., Prévost S. (éds), Congrès Mondial de Linguistique Française -, p. 723-744. DOI 10.1051/cmlf/2010076 - 2011, «Quelles sont les significations dessinées par le dialogisme ? Une perspective en analyse de discours ", dans J. Bres, A. Nowakowska, J.M. Sarale, S. Sarrazin (coord.) Actes du colloque international Dialogisme : langue, discours (8-10 septembre 2010, Montpellier), mis en ligne le 10 juillet 2011.

- 2015, « Analyse discursive de points de vue dans des récits de parcours professionnels extraits d'entretiens », dans M. Carcassonne, D. Cunha, C. Donahue, F. François, A. Rabatel, 2015, Points de vue sur le point de vue, Limoges, Lambert-Lucas, pp. 77-167.

Carcassonne M., Salazar-Orvig A., Bensalah A., 2001, « Des récits dans des entretiens de recherche : entre narration et interprétation », dans Revue québécoise de linguistique, vol.29, $\mathrm{n}^{\circ} 1$, pp. 97-122. http://id.erudit.org/iderudit/039431ar

Carcassonne M., Servel L., 2009, « Dire le temps, dire le changement » dans Temporalités [En ligne], 10 | 2009, mis en ligne le 30 novembre 2009, URL : http://temporalites.revues.org/ index1122.html

Chareaudeau P., Maingueneau D., 2002, Dictionnaire d'analyse du discours, Paris, Seuil.

Dubar C., 1991, La socialisation. Construction des identités sociales et professionnelles, Paris, A. Colin, 1991.

Duméry H., « TEMPORALITÉ », Encyclopcedia Universalis [en ligne], consulté le 20 janvier 2017. URL : http://www.universalis.fr/encyclopedie/temporalite/

Durkheim E., 1968 [1912], Les formes élémentaires de la vie religieuse, Paris, P.U.F.

François Frédéric, 1982, «Ebauches d'une dialogique », dans Connexions, n³8, p. 61-87

- 1984, « Conduite du dialogue » dans François F., Hudelot C., Sabeau-Jouannet E., Conduites

linguistiques chez le jeune enfant, Paris, PUH, p. 117-183.

- 1994, Morale et mises en mots, Paris, L'Harmattan. 
- 2004, Enfants et récits, mise en mots et « reste »-Textes classés et présentés par R. Delamotte Legrand, Paris, Presses Universitaires du Septentrion.

- 2006, Rêves, récits de rêves et autres textes. Un essai sur la lecture comme expérience indirecte. Paris, Lambert-Lucas.

- 2009, Essais sur quelques figures de l'orientation ; hétérogénéités, mouvements et styles. Paris, LambertLucas.

- 2012, Bakhtine tout nu, Limoges, Lambert-Lucas.

- 2014, « Avant-propos. Le « dialogisme» ? Ou plutôt « quelques figures du dialogue, leurs communautés et leurs différences, un point de vue ", Ela. Études de linguistique appliquée 2014/1 (n 173), p. 17-26.

Gardin B., 1978, « Volochinov ou Bakhtine? », dans La pensée n 197, pp. 87-100

Genette G., 1972, « Discours du récit » dans Figures III, Paris, Seuil, p. 65-282.

- 1983, Nouveau discours du récit, Paris, Seuil.

Greimas A. J., 1966, « Éléments pour une théorie de l'interprétation du récit mythique ", dans Communications, 8, Recherches sémiologiques : l'analyse structurale du récit, p. 28-59.

Doi : 10.3406/comm.1966.1114, http://www.persee.fr/doc/

comm_0588-8018_1966_num_8_1_1114

Greimas A.J., Fontanille J., 1991, «L'épistémologie des passions » dans Sémiotique des passions, Seuil, Paris.

Gülich E., Quasthoff U., 1985, « Narrative analysis », dans Teun A. Van Dijk (dir.), Handbook of Discourse Analysis, London, Academic Press, t. 2, p. 169-192.

Gurvitch G., 1958, « La multiplicité des temps sociaux », Les cours de la Sorbonne, Paris, Centre de documentation universitaire.

Grossmann F., Tutin A., 2005a, « Sur la prédicativité des adjectifs », Cahiers de Lexicologie, 86, 2005-1, p. 1-18

- 2005b, « Présentation », Lidil, 32, Sémantique des noms et adjectifs d'émotion, p. 1-20.

Halbwachs M., 1950, La mémoire collective, Paris, PUF.

Hébert L., 2006, « Le modèle actantiel », dans Louis Hébert (dir.), Signo [en ligne], Rimouski (Québec), http://www.signosemio.com/greimas/modele-actantiel.asp.

Heidegger M., 1964 [1927]), L'être et le temps, Paris, Gallimard.

Husserl E., 1992 [1905]) Les leçons pour une phénoménologie de la conscience intime du temps, Paris, PUF.

Ivanova I., 2003, Le dialogue dans la linguistique soviétique des années 1920-1930. Cahiers de l'Institut de linguistique et des sciences du langage (Université de Lausanne), 14, 157-182.

Kerbrat-Orecchioni C., 1980, L'énonciation, de la subjectivité dans le langage, Paris. A. Colin

Kaufmann J.C., 2004, L'invention de soi, une théorie de l'identité, Paris, Armand Colin.

Kristeva J., 1967, « Bakhtine, le mot, le dialogue, le roman », Critique, avril, nº 239 : 434-443.

Kundera M., 1993, Les testaments trahis, Paris, Gallimard (Coll. Folio).

Labov W., 1978 [1972], « La transformation du vécu à travers la syntaxe narrative », dans Le parler ordinaire, chap. 9, p. 289-335, Paris, Minuit.

- 1997, « Some further steps in narrative analysis » dans Journal of narrative and life history 7, p. 395-415. 
- 2007, « Narrative pre-construction » dans M. Bamberg (ed.), Narrative - state of the art. Amsterdam, Philadelphia : John Benjamins, p. 47-56.

Laforest M. (dir.), 1996, Autour de la narration : les abords du récit conversationnel, Québec, Nuit Blanche.

Larivaille P. 1974, «L'analyse (morpho)logique du récit », Poétique, n 19, p. 368-388

Merleau-Ponty M., 1947, « La temporalité » in Phénoménologie de la perception, Paris, Gallimard.

Moirand, S., 2003, Quelles catégories descriptives pour la mise au jour des genres du discours ?. In Contributions à la journée organisée par Kerbrat-Orecchioni C. \& Traverso, V. le 18 avril 2003, Université Lumière Lyon, http://icar.univ-lyon2.fr/Equipe1/actes/journees_genre.htm

Mondada L., 2001, « L'entretien comme événement interactionnel » dans M. Grosjean J.P. Thibaud (eds), L'espace urbain en méthodes. Marseille, Parenthèse, p. 197-214

Nossik N., 2011, « Les récits de vie comme corpus sociolinguistique : une approche discursive et interactionnelle », Corpus, 10, 119-135.

- 2012, «Sur l'ouvrage de Jean-Paul Bronckart et Cristian Bota, 2011, Bakhtine démasqué. Histoire d'un menteur, d'une escroquerie et d'un délire collectif, Genève, Droz, 629 pages », Semen [En ligne], 33 | 2012, mis en ligne le 04 juin 2012, consulté le 11 mars 2013. URL : http://semen.revues.org/9537

Pollack M., 1993, «L'entretien en sociologie », Site de l'Institut d'histoire du temps présent, http://www.ihtp.cnrs.fr/spip.php\%3Farticle239\&lang=fr.html

Ochs E., Capps L., 2001, Living narrative : creative lives in every day storytelling, Havard University Press, Cambridge.

Ochs E., 2004, « Narrative lessons », dans Duranti A. (ed), A Companion to Linguistic Anthropology, Oxford, Blackwell, p. 269-289. Traduction française dans Elinor Ochs, 2014, « Ce que les récits nous apprennent ", Semen [En ligne], 37.

Penrose R., 2013, Les cycles du temps, une nouvelle vision de l'univers, Odile Jacob.

Propp V., [1928] 1965, Morphologie du conte, Paris, Seuil.

Rabatel A., 1997, Une Histoire du point de vue, Metz, CELTED.

- 1998, La Construction textuelle du point de vue, Lausanne, Delachaux \& Niestlé.

- 2003, « Pour une narratologie énonciative ou pour une approche énonciative de la narration » dans Vox Poetica, http://www.vox-poetica.org/t/lna/rabatel.htm

- 2008, Homo narrans. Pour une analyse énonciative et interactionnelle du récit, Limoges, LambertLucas.

- 2015, «Retour sur un parcours en énonciation », dans Carcassonne M., Cunha D., Donahue, C., François F., Rabatel A., Point de vue sur le point de vue, Limoges, Lambert-Lucas, p. 327-355.

Revaz Françoise, Fillettaz Laurent, 1996, « Actualités du récit dans le champ de la linguistique des discours oraux : le cas des narrations en situation d'entretien ", Protée, Volume 34, numéro 2-3, p. 53-66.

Revaz Françoise, 1997, « Le récit dans la presse écrite », dans Pratiques 94, p. 19-33.

Ricœur Paul, 1983, Temps et récit, tome 1: L'intrigue et le récit historique, Paris, Seuil.

- 1984, Temps et récit, tome $2:$ La configuration dans le récit de fiction, Paris, Seuil.

- 1985, Temps et récit, tome 3 : Le temps raconté, Paris, Seuil.

- 1990, Soi-même comme un autre, Paris, Editions du Seuil

Roulet E., 1980, « Echanges, interventions et actes de langage dans la structure de la conversation », dans Etudes de linguistique appliquée n 44, p. 7-39. 
Sacks H., 1995 [1992], Lectures on conversation; vol 1 et 2, ed. by G. Jefferson, with an introduction by E.A. Schegloff. Oxford : Bruxelles.

Salazar Orvig, A., 1999, Les mouvements du discours. Style, référence et dialogue dans des entretiens cliniques. Paris, L’Harmattan.

Sainsaulieu R., 1977, L'identité au travail, Paris, Presses Nationales de la Fondation des Sciences Politiques

Schegloff E., 1968, « Sequencing in conversationnel openings », dans American Anthropologist 70, 4 : 1075-1095.

Sériot P., «Préface. Volochinov, la philosophie de l'enthymème et la double nature du signe ", dans Volochinov V., Marxisme et philosophie du langage, Limoges, Lambert-Lucas, 2010, pp. 13-109.

Stern D., 1989, « Le sens d'un soi émergent » in Le monde interpersonnel du nourrisson, pp. 57-95, Paris, Presses Universitaires de France.

Todorov T., 1968, « La grammaire du récit ». In: Langages, $3^{\mathrm{e}}$ année, $\mathrm{n}^{\circ} 12$, 1968. Linguistique et littérature. pp.94-102.doi :10.3406/lgge.1968.2355 ; http://www.persee.fr/doc/

lgge_0458-726x_1968_num_3_12_2355

- 1981, Mikhail Bakhtine, le principe dialogique, Paris, Seuil.

Tylkowski I., 2012, V. N. Volosinov en contexte, essai d'épistémologie historique, Limoges, LambertLucas.

Vion R., 1992, La communication verbale, analyse des interactions, Paris, Hachette.

Volochinov N. (publié sous le nom de Bakhtine), 1977 [1929], Marxisme et philosophie du langage. Essai d'application de la méthode sociologique en linguistique, traduction et présentation par Marina Yaguelo, Paris, les Editions de Minuit

Yocaris I., Zemmour D., 2010, «Qu'est-ce que la fonction cubiste ? La "construction textuelle du point de vue" » dans l'Herbe et La Route des Flandres de Claude-Simon dans F. Neveu F., V. Muni Toke, T. Klinger, J. Durand, L. Mondada, S. Prévost (éds), Congrès Mondial de Linguistique Française - CMLF 2010, p. 1237- 1263. DOI 10.1051/cmlf/2010086

\section{APPENDIXES}

\section{Annexe 1 - Guide d'entretien}

1- Pouvez-vous me raconter en quelques minutes votre parcours?

2a- Comment en êtes-vous arrivé à choisir cette voie ? 2b- qu'est-ce qui vous attirait le plus au départ dans ce métier

3a- Comment en êtes-vous arrivé occuper ce poste ? 3b- Pensez-vous évoluer par rapport à votre poste actuel?

4a- Quelle formation avez-vous suivie initialement ? 4b- Feriez-vous le même choix de formation aujourd'hui ? 4c- Suivez-vous aussi des compléments de formation dans le cadre de la formation continue? Quels en sont les thèmes?

5- Quels sont les avantages et inconvénients de votre emploi actuel?

6a- Pouvez-vous me décrire une journée type de travail ? 6b- de vacances? 
7- Comment est organisée votre semaine en termes de vie professionnelle, familiale, sportive, de loisirs, associatives, etc. ?

8a- Aimeriez-vous aujourd'hui pouvoir changer la façon dont les choses se passent dans votre vie professionnelle? 8b-Comment ? 8c- Feriez-vous le même choix de métier aujourd'hui ? 8d- Pourquoi?

9- si vous deviez recruter un collègue pour travailler avec vous, quels aspects prendriezvous plus particulièrement en compte?

10- Quels conseils ou mises en garde donneriez-vous à quelqu'un qui voudrait suivre la même voie que vous?

\section{Annexe 2 - Caractéristiques de passation des entretiens cités}

\begin{tabular}{|l|l|l|}
\hline$N^{\circ}$ entretien & Initiales des interviewés $(\mathrm{H}, \mathrm{F}$, âge $)$ & Initiales des intervieweurs-étudiants $(\mathrm{H}, \mathrm{F})$ \\
\hline 1 & A, homme, 50 ans & Z et $\mathrm{H}$, hommes \\
\hline 2 & B, homme, 60 ans & X et $\mathrm{Y}$, hommes \\
\hline 3 & C, homme, 50 ans & W femme \\
\hline 4 & D, homme 53 ans & K et $\mathrm{V}$, femmes \\
\hline
\end{tabular}

\section{Annexe 3 - Conventions de transcription}

\begin{tabular}{|l|l|}
\hline$/$ & respiration intonative (prise de souffle supérieure à 20 centisecondes) \\
\hline$?$ & Question \\
\hline$::$ & allongement de syllabe \\
\hline traditionNEL & syllabe appuyée \\
\hline xxxx & incompréhensible (un x par seconde) \\
\hline$(2$ sec) & pause de deux secondes (une + par seconde) \\
\hline$<$ croise les bras & $\begin{array}{l}\text { entre chevrons et en italique : commentaire sur le non verbal (gestes, mimiques) } \\
\text { ou sur les événements extérieurs }\end{array}$ \\
\hline$>$ & $\begin{array}{l}\text { en gras : phatème ou intervention de l'autre interlocuteur, ne constituant pas à } \\
\text { proprement parler un tour de parole, d'où son insertion dans le tour de parole } \\
\text { du locuteur en train de parler }\end{array}$ \\
\hline mmh & \begin{tabular}{l} 
coupure de mot coupure de mot ou de phrase \\
\hline
\end{tabular}
\end{tabular}




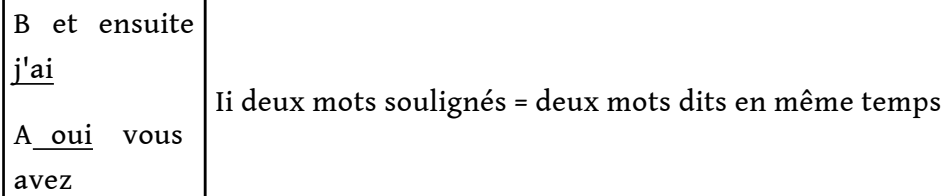

\section{Annexe 4 - Premier extrait de l'entretien de D (récit initial du parcours)}

\begin{tabular}{|c|c|c|}
\hline K1 & 1 & et hum / par la suite comment vous êtes-vous retrouvé à diriger ce cabinet? \\
\hline D7 & 1 & alors je vous explique euh / \\
\hline & 2 & enthousiaste et jeune / euh parce que j'avais 26 ans euh $(2 \mathrm{sec})$ \\
\hline & 3 & beaucoup de clients::: dans le cabinet où je faisais le stage m'appréciaient / \\
\hline & 4 & et à force d'avoir un tout petit peu de relationnel \\
\hline & 5 & $\begin{array}{l}\text { je suis arrivé à apporter à mon cabinet où je faisais mon stage d'expert-comptable } \\
\text { une cinquantaine de clients / }\end{array}$ \\
\hline & 6 & les clients aimaient beaucoup la manière dont j'essayais de résoudre leurs problèmes / \\
\hline & 7 & $\begin{array}{l}\text { dans la mesure où pour moi un expert-comptable et un commissaire aux comptes est } \\
\text { déjà un docteur / }\end{array}$ \\
\hline & 8 & un docteur c'est l'homme qui apporte quelque chose / \\
\hline & 9 & il touche de l'argent / mais l'argent il l'aura toujours / il a des études / \\
\hline & 10 & mais le fait d'apporter une puissance une force / une connaissance / \\
\hline & 11 & $\begin{array}{l}\text { pour aiGUIller / un peu euh des gens qui réussissent mais qui s'améliorent est } \\
\text { toujours un élément en plus / }\end{array}$ \\
\hline & 12 & $\begin{array}{l}\text { et donc ce qui s'est passé c'est que dans ce cabinet beaucoup de gens sont venus } \\
\text { grâce à moi / }\end{array}$ \\
\hline & 13 & mais je ne faisais que mon stage / \\
\hline & 14 & et c'est vrai qu'on m'a proposé d'être associé mais euh dans ce cabinet où j'étais \\
\hline & 15 & euh je trouve qu'ils avaient un peu une vue à court terme / \\
\hline & 16 & ce qui les intéressaient le plus c'était la facturation et l'argent / \\
\hline & 17 & moi ce qui m’intéressait le plus c'était plutôt apporter et évoluer vers du long terme / \\
\hline & 18 & donc euh quand j'ai terminé mon stage / \\
\hline
\end{tabular}




\begin{tabular}{|c|c|}
\hline 19 & euh ben on m'a dit voilà on vous associe / \\
\hline 20 & et j'ai dit non / j'ai dit non / je vais créer mon entreprise / \\
\hline 21 & j'avais quel âge ? 27 ans / \\
\hline 22 & donc à 27 ans j'ai dit \\
\hline 23 & ben après tout tu as des clients qui t'apprécient / \\
\hline 24 & tu es compétent / \\
\hline 25 & tu as eu une bonne formation grâce à l'université / \\
\hline 26 & tu as eu une chance inouïe d'avoir eu de très bons professeurs qui t'ont formé \\
\hline 27 & après tout ben tu te débrouilles / \\
\hline 28 & donc c'est là où j'ai plutôt opté pour créer mon cabinet / \\
\hline 29 & $\begin{array}{l}\text { d'autant plus que euh le cabinet où je faisais mon stage d'expert-comptable était } \\
\text { dans le XXXème (un arrondissement populaire de Paris) / }\end{array}$ \\
\hline 30 & $\begin{array}{l}\text { et que j'avais l'opportunité euh d'avoir des locaux::: dans le XXX à XXXX } \\
\text { (un beau quartier de Paris) / }\end{array}$ \\
\hline 31 & $\begin{array}{l}\text { et donc je me suis dit ben non vaut mieux commencer à zéro sans être assuré } \\
\text { d'une rémunération / }\end{array}$ \\
\hline 32 & de toutes façons étant simple de nature et aimant mon métier / \\
\hline 33 & si demain j'avais pas d'argent je travaillerais / \\
\hline 34 & donc euh ça n'a pas été l'argent qui a motivé la création de mon cabinet / \\
\hline 35 & c'était la volonté de développer / d'apporter / \\
\hline 36 & et l'argent est arrivé par la suite / \\
\hline 37 & parce que l'argent n'est pas l'élément déterminant / \\
\hline 38 & $\begin{array}{l}\text { ce qui est déterminant dans la vie c'est toujours une volonté de } \\
\text { vouloir réussir / }(2 \mathrm{sec})\end{array}$ \\
\hline 39 & donc c'est comme ça ( $1 \mathrm{sec})$ très novice j'ai fait mon cabinet / \\
\hline 40 & et à l'origine je n'avais qu'un petit bureau ( $1 \mathrm{sec})$ de $17 \mathrm{~m} 2$ non même pas de::: $6 \mathrm{~m} 2$ / \\
\hline 41 & et euh au fur et à mesure du développement j'ai pu embaucher du monde / \\
\hline 42 & et donc il a été nécessaire d'avoir des locaux plus grands / \\
\hline
\end{tabular}




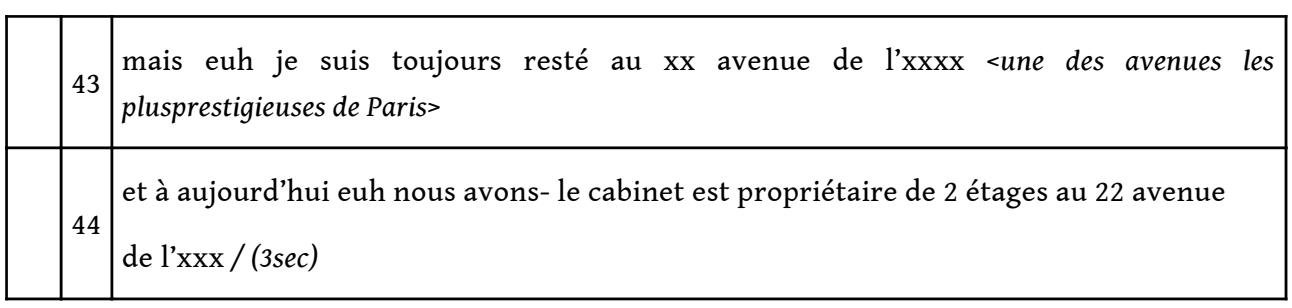

\section{Commentaire linéaire de l'extrait du récit (D71-44) de D}

On peut interpréter ce récit avec une grille de lecture structuraliste, tout en faisant un lien avec la notion de chronotope : on y repère cinq étapes ainsi qu'un certain nombre $d^{\prime}$ '« opposants » et d' " adjuvants »; on y repère également un mouvement discursif de chonotopes opposant deux espaces, deux périodes, et deux positionnements affectivoidéologiques très contrastés :

i) L'étape 1 (1.1-13 puis 15-17) décrit une situation initiale relativement stable où les clients, appréciant l'aide apportée par le personnage $\mathrm{D}$, sont de de ce fait de plus en plus en nombreux ; ce qu'apprécient à leur tour les supérieurs de D.

Au cours de cette étape, le narrateur omet de dire que tout n'est pas aussi rose que ne le laisse entendre sa description : en effet, on apprend plus tard (1. 15-17) que D n'était pas en accord avec les valeurs de ses supérieurs et jugeait son activité de stagiaire peu intéressante (car limitée à la facturation dans une vue à court terme). On repère donc ici un manque (au sens de la théorie structuraliste qui l'a décrit comme étant en général « déclencheur » d'une action, et qui peut aussi s'interpréter comme un aspect temporel affectivo-idéologique caractérisant le cabinet dans lequel D a fait son stage). Ce manque va déclencher chez $\mathrm{D}$ la décision de ne pas accepter l'offre d'embauche de ses supérieurs. Ce refus crée un effet (relatif) de surprise sur le récepteur, car le narrateur a retardé la livraison d'une information importante (narration « réticente » produisant un effet « intrigant » au sens de Baroni, 2009).

ii) L'étape 2 (force perturbatrice, 1.14 puis 18-19) correspond au moment où les supérieurs de D lui proposent de devenir leur associé : cette proposition peut être vue comme une embûche sur le parcours de $\mathrm{D}$ et les supérieurs comme des opposants à son projet de vie.

iii) L'étape 3 (état de déséquilibre, 1. 20-22) correspond au refus de D d'être associé, et à son choix plus « risqué » de créer son propre cabinet. La " prise de risque », la " vue à long terme », « l'aide ou l'apport » en tant qu'expert sont ici des valeurs mises en avant par D et sont présentées comme supérieures à celles mises en avant par ses supérieurs (gain d'argent à court terme).

iv) L'étape 4 (force équilibrante, 1. 23-35) correspond à la délibération amenant $\mathrm{D}$ à prendre la décision de créer son propre cabinet, et à refuser la proposition de ses supérieurs. D insiste plus sur certains adjuvants que sur d'autres dans cette délibération : l'adjuvant « formation » est très explicité (1.25-27) tandis que l'adjuvant « avoir des locaux » (1. 30) est mentionné de manière beaucoup plus allusive (on ne sait pas grâce à qui).

v) L'étape 5 (1. 36-44) correspond au retour à l'équilibre : en suivant ses propres valeurs, D a créé un cabinet qui a prospéré très vite (1. 36-44).

Annexe 5- Deuxième extrait de l'entretien de $\mathrm{D}$ reconfigurant à distance l'interprétation de l'extrait de l'annexe 4 


\begin{tabular}{|c|c|c|}
\hline V8 & 1 & alors maintenant on va vous demander / \\
\hline V8 & 2 & euh quels sont les avantages et les inconvénients de votre emploi actuel / \\
\hline D11 & 1 & hum (2sec) comme tout emploi qui nécessite une passion / \\
\hline D11 & 2 & et on la retrouve dans beaucoup de milieux / \\
\hline D11 & 3 & euh malheureusement on doit sacrifier certaines choses / $(2 \mathrm{sec})$ \\
\hline D11 & 4 & euh mais on doit la gérer à travers le temps / \\
\hline D11 & 5 & $\begin{array}{l}\text { euh là les inconvénients euh c'est vrai que c'est un métier passionné / passionnant / } \\
\text { euh humm ( } 1 \mathrm{sec})\end{array}$ \\
\hline D11 & 6 & je dirais euh (1 sec) malheureusement c'est un peu une vie familiale / \\
\hline D11 & 7 & $\begin{array}{l}\text { toutes les professions libérales euh où les gens sont très pris humm / et::: tout le } \\
\text { monde vous le dira / }\end{array}$ \\
\hline D11 & 8 & euh c'est un peu au détriment d'une vie familiale / \\
\hline D11 & 9 & y a pas de solution miracle / c'est comme ça / $(2 \mathrm{sec})$ \\
\hline D11 & 10 & euh c'est l'inconvénient le plus important / \\
\hline D11 & 11 & $\begin{array}{l}\text { mais EUh c'est une chose...je dirais que c'est quelque chose qui peut être géré et qui se } \\
\text { gère / }\end{array}$ \\
\hline D11 & 12 & euh c'est l'un des inconvénients mais sinon euh on ne voit que des avantages / \\
\hline D11 & 13 & la découverte de beaucoup de métiers / \\
\hline D11 & 14 & le fait de voyager / dans le monde entier / \\
\hline D11 & 15 & le fait d'avoir des contacts / \\
\hline D11 & 16 & le fait de s'enrichir quotidiennement \\
\hline D11 & 17 & et le fait de découvrir / ( $2 \mathrm{sec})$ \\
\hline D11 & 18 & euh en ce qui concerne je dirais l'aspect euh rémunération ou capital / (3 sec) \\
\hline D11 & 19 & $\begin{array}{l}\text { c'est un aspect où en général un::: expert comptable / un commissaire aux comptes / } \\
\text { euh je dirais / }\end{array}$ \\
\hline D11 & 20 & ses revenus normaux annuels sont de 150000 euros / \\
\hline D11 & 21 & donc euh je dirais euh l'argent n'est pas un élément euh / \\
\hline D11 & 22 & mais c'est cet aspect de la vie familiale qui est un peu::: sacrifié / \\
\hline
\end{tabular}




\begin{tabular}{|l|l|l|}
\hline D11 & 23 & $\begin{array}{l}\text { mais on le retrouve chez les notaires / chez les avocats / chez les::: hommes chefs } \\
\text { d'entreprises / }\end{array}$ \\
\hline D11 & 24 & simplement il faut être organisé / \\
\hline D11 & 25 & c'est cet aspect qui est un peu::: gênant \\
\hline & & \\
\hline
\end{tabular}

\section{Annexe 6 : premier extrait de l'entretien de Monsieur A}

\begin{tabular}{|c|c|c|}
\hline A2 & 17 & c'est aussi un métier qui permet d'être indépendant <vibration du téléphone> / \\
\hline A2 & 18 & il y a pas beaucoup de métiers qui permettent d'être indépendANT / \\
\hline A2 & 19 & et donc euh c'est ça aussi qui m'a attiré / \\
\hline A2 & 20 & $\begin{array}{l}\text { je::: savais comme je l'ai dit tout à l'heure que depuis le début je voulais exercer ce } \\
\text { métier en étant libéral euh / }\end{array}$ \\
\hline A2 & 21 & mais parce que voilà c'est aussi une question de caractère euh \\
\hline A2 & 22 & moi je :::sen- pensais que que je me sentirais mieux en étant MAItre de mon travail \\
\hline A2 & 23 & euh plutôt que de recevoir des ordres / \\
\hline A2 & 24 & $\begin{array}{l}\text { et aussi parce que c'est toujours plus:::plus agréable d'avoir les dossiers d'une manière::: } \\
\text { / }\end{array}$ \\
\hline A2 & 25 & plutôt que que d'être un peu en quelque sorte comme euh comme à la chaine / \\
\hline A2 & 26 & enfin de de traiter les dossiers du début jusqu'à la fin / \\
\hline A2 & 27 & $\begin{array}{l}\text { donc quand on est dans un grand cabinet d'audit/ on se spé- on a rapidement tendance } \\
\text { à se spécialiser / }\end{array}$ \\
\hline A2 & 28 & $\begin{array}{l}\text { et une fois que l'on s'est spécialisé euh c'est assez difficile euh:::de de faire d'autres } \\
\text { choses / }\end{array}$ \\
\hline A2 & 29 & euh::: ça permet aussi de gérer son emploi du temps comme on veut / \\
\hline A2 & 30 & euh:::moi j’ai toujours eu un peu de mal avec la hiérarchie / \\
\hline
\end{tabular}

\section{Annexe 7 : deuxième extrait de l'entretien de A}

\begin{tabular}{|l|l|l|}
\hline A7 & 29 & le plus gros inconvénient / par contre euh \\
\hline A7 & 30 & c'est la contrepartie je pense de tous les métiers passionnants \\
\hline A7 & 31 & c'est que euh::: on travaille beaucoup on travaille énormément / \\
\hline
\end{tabular}




\begin{tabular}{|l|l|l|}
\hline A7 & 32 & euh mais si on veut à la fois faire des choses intéressantes \\
\hline A7 & 33 & $\begin{array}{l}\text { et si on veut à la fois euh gagner un peu d'argent et si on/ euh:::veut avoir euh:::un } \\
\text { travail euh::: agréable }\end{array}$ \\
\hline A7 & 34 & ben il faut travailler beaucoup c'est normal / \\
\hline A7 & 35 & mais c'est vrai que c'est c'est c'est le principal inconvénient \\
\hline
\end{tabular}

\section{Annexe 8 : extrait de l'entretien de B}

\begin{tabular}{|l|l|l|}
\hline B2 & 81 & finalement être indépendant on travaille peut-être plus \\
\hline B2 & 82 & mais au moins on a plus de:::on a plus de plus marges de manœuvres \\
\hline B2 & 83 & et surtout une beaucoup plus grande de liberté / bon \\
\hline B2 & 84 & <lit la grille d'entretien> <bon avantage et inconvénient de l'emploi> \\
\hline B2 & 85 & c'est qu'y a des horaires de travail qui sont complètement déments / \\
\hline B2 & 86 & -fin je sais pas ton père- moi c'est encore pire que lui j'pense \\
\hline B2 & 87 & $\begin{array}{l}\text { c'est au moins 10-12 heures par jour euh une partie du week-end aussi } \\
\text { / }\end{array}$ \\
\hline B2 & 88 & euh les vacances euh c'est- quand on peut prendre \\
\hline B2 & 89 & quatre semaines l'été c'est formidable \\
\hline
\end{tabular}

\section{Annexe 9 : extrait de l'entretien de C}

\section{NOTES}

1. Et non comme un outil qui mènerait indirectement vers un autre objet de connaissance ; cela dit, analyser le fonctionnement narratif d'un même corpus d'entretiens permet de caractériser les pratiques narratives d'un groupe d'interviewés, et donc d'en dire plus que sur le seul fonctionnement de l'entretien biographique.

2. Cf. annexes 1 et 2 pour le guide d'entretien et les caractéristiques de passation des entretiens mobilisés ici.

3. Le recueil puis l'analyse des entretiens (selon des axes qui ne sont pas ceux présentés ici) a en effet amené les étudiants à se centrer (i) sur la façon dont les EC se présentent spontanément en mettant avant certains aspects de leurs parcours ou de leur métier plus que d'autres, (ii) sur la façon dont ils peuvent effacer ou au contraire engager fortement leur subjectivité énonciative selon les thèmes abordés.

4. Les thèses initiales de Volochinov ont été rendues accessibles en France sous le nom de Bakhtine, d'abord par un article de Kristeva (1967), ensuite par un ouvrage publié en russe en 1929 et traduit en français en 1977 (Le marxisme et la philosophie du langage), enfin par un ouvrage 
de Todorov présentant un certain nombre de traductions (1981). Volochinov a ensuite été repéré comme étant l'auteur de Marxisme et philosophie du langage par un certain nombre de linguistes ; cf. entre autres Gardin 1978 ; Todorov 1981, Ivanova 2003, Moirand 2003, Tylkowski 2012, Sériot (in Volochinov $2010: 13-95)$. Cette dernière référence concerne la préface très documentée précédant la retraduction de Marxisme et philosophie du langage par Sériot et Ageeva (2010), publié cette fois-ci sous le nom de Volochinov. D'autres chercheurs ont par ailleurs souligné le fait qu'un certain nombre d'autres écrits signés par Bakhtine lui revenaient effectivement : cf. entre autres François 2012, Nossik 2012 ainsi que l'ensemble des auteurs à qui répondent J.P. Bronckart et C. Bota (2014) suite aux critiques suscitées par leur ouvrage paru en 2011.

5. «Toute énonciation, même sous sa forme écrite figée, est une réponse à quelque chose et est construite comme telle. Elle n'est qu'un maillon de la chaîne des actes de parole. Toute inscription prolonge celles qui l'ont précédées, engage une polémique avec elles, s'attend à des réactions actives de compréhension, anticipe sur celles-ci, etc...» (Volochinov, 1977 [1929]: 105-106).

6. «L'œuvre prédétermine les positions responsives de l'autre dans les conditions complexes de l'échange verbal d'une sphère culturelle donnée. L'œuvre est un maillon dans la chaîne de l'échange verbal; semblable à la réplique du dialogue, elle se rattache aux autres œuvresénoncés : à celles auxquelles elle répond et à celles qui lui répondent, et dans le même temps, semblable en cela à la réplique du dialogue, elle en est séparée par la frontière absolue de l'alternance des sujets parlants ». (Bakhtine, 1984 [1952-53] : 282).

7. Rabatel a récemment souligné qu'un certain nombre d'auteurs ont, à la suite de Bakhtine, analysé le dialogisme et l'hétérogénéité énonciative en se centrant surtout sur « les autres que soi (bref, les autres au sens trivial du terme)»; mais qu'une forme d'hétérogénéité complémentaire concerne « les autres de moi, les autres moi-mêmes, un rapport opaque de soi à soi » $(2015: 344)$.

8. Cf. les textes publiés entre 1988 et 2001 et rassemblés par R. Delamotte-Legrand dans F. François 2004.

9. Prenons pour exemple ces deux citations (datant respectivement de 1994 et 2014) qui précisent la notion de mouvement discursif et interprétatif (les italiques sont de nous) :

- « Le mouvement dans la relation du discours de l'un au discours de l'autre ou du discours sur soi au discours sur soi constituerait alors la catégorie centrale de l'analyse des énoncés par opposition aux phrases de la grammaire [...]. Un énoncé, soit explicitement soit contextuellement prétend à une certaine réponse. Mais parmi tout ce qui peut être dit, la réponse déplace le cadre discursif » (François, $1994: 52)$;

- «Qu'il s'agisse d'un dialogue verbal entre deux personnes, du dialogue corporel d'un échange de sourires, du dialogue avec soi où on revient sur ce qu'on vient de dire ou de se dire, de faire ou de ressentir, il y a bien un mouvement, un déplacement par rapport à ce qui précède » (François, 2014 : 19).

10. Notons que ce rapport de soi à soi se complique par le fait que « le discours que l'on tient sur soi est souvent intermédiaire entre le discours objectif de l'autre et le discours subjectif de soi : tenir un discours sur soi met en effet le locuteur dans la situation d'un quasi-autre qui n'est pas un vrai autre. Toute personne peut parler d'elle en reprenant à son propre compte ce qu'autrui a dit d'elle (par exemple ce qu'on lui a raconté d'elle étant enfant, ce que les médecins disent d'elle) tout en indiquant simultanément ou alternativement ce qu'elle connaît d'elle par familiarité (par exemple la perception qu'elle a de son propre caractère, de son rapport à autrui, mais aussi ses craintes, ses espoirs, etc.) " (Carcassonne, $2010: 725$ ).

11. Selon ces champs, la temporalité renvoie à des aspects très différents : temporalité réversible des physiciens les amenant à dire que la distinction entre passé, présent et futur n'est qu'une illusion (Einstein), temporalité cyclique des mathématiciens (Penrose 2013), temporalité comme simple ordre d'antériorité ou de postériorité de certains philosophes (par ex. Aristote ou 
Leibniz), temporalité nécessairement reliée à une conscience qui «l'éprouve » chez d'autres philosophes, temporalités sociales (construites socio-culturellement par les groupes auxquels on appartient (temps pour l'autre) et déterminant le temps biographique (temps pour soi) chez les sociologues (entre autres Durkheim 1968 [1912], Gurvitch 1958), temps linguistique comme marqué essentiellement par les temps et aspects verbaux et se différenciant fortement du temps physique et du temps chronique (Benveniste 1974), etc.

12. Pour un tour d'horizon concernant la notion de temporalité affective en phénoménologie, cf. ALTER-Revue de phénoménologie n`3, Temporalité et affection, 1994.

13. Ce que D. Stern (qui n'est pas un sémanticien mais un psychologue inspiré par la perspective phénoménologique) a nommé «affect de vitalité » (1989: 78) : par exemple, on peut faire un sourire "explosif» (premier cas) ou se lever de sa chaise de manière "explosive » (deuxième cas). Dans le premier cas l'affect de vitalité (explosion) est associé à un affect catégoriel (sourire, joie), dans le second pas nécessairement.

14. cf. l'article de H. Duméry définissant la «temporalité » dans l'Encyclopaedia Universalis en ligne.

15. Les différences entre ces termes ne sont pas fondamentales pour notre propos.

16. Nous avons insisté dans la partie 1.1 sur la dimension dialogique et interprétative de la notion de «mouvement discursif», et sur la façon dont cette notion s'inspire des écrits de V. N. Volochinov et Bakhtine puis de F. François.

17. M. Kundera nomme «sens abstrait » cette dimension affective » dont on se souvient: « Les situations les plus chères, les plus importantes, sont perdues à jamais. Ce qu'il en reste, c'est leur sens abstrait (j'ai défendu ce point de vue, lui tel autre, j'ai été agressif, lui défensif » (1993: 156). Notons qu'il est à l'inverse possible de se souvenir d'une vérité narrative en perdant l'affect, ce qui est également une forme de temporalité affective.

18. Il existe aujourd'hui des cousins non francophones : W. Booth, F. Stanzel et leurs continuateurs M. Bal, S. Seymour.

19. Comme le résume L. Hébert (2006, en ligne), «1) Le sujet (par exemple, le prince) est ce qui veut ou ne veut pas être conjoint à 2) un objet (par exemple, la princesse délivrée), 3) Le destinateur (par exemple, le roi) est ce qui incite à faire l'action, alors que 4) le destinataire (par exemple, la princesse) est ce qui en bénéficiera. Enfin, 5) un adjuvant (par exemple, l'épée magique, le cheval, le courage du prince) aide à la réalisation de l'action, tandis qu'un 6) opposant (par exemple, la sorcière, le dragon, la fatigue du prince et un soupçon de terreur) y nuit ».

20. Les italiques sont de nous et signalent les 5 étapes : 1 situation stable, 2 force perturbatrice, 3 état de déséquilibre, 4 force inverse, 5 équilibre nouveau. Les forces assurent donc la «transformation d'un état en un autre ».

21. F. Revaz précise qu'outre la tension, un « récit» ne peut s'appeler "récit» que si les «motifs » des actions entre le nœud et le dénouement sont explicités, ces deux dimensions étant le véritable ressort de toute « mise en intrigue » (Ricœur), cf. infra III.

22. Cette approche permet à R. Baroni de distinguer « les actes intentionnels de l'intrigant - celui qui raconte de manière réticente afin d'intriguer les destinataires du récit - et de l'intrigué - celui qui face à cette réticence, se prend au jeu de l'intrigant et tente de répondre à la question “comment cette histoire se finira-t-elle"? » (Ibid. : 11). Les termes d' « intrigant » et d' « intrigué » sont empruntés à J. Villeneuve, Le sens de l'intrigue, Québec, Presse de l'université Laval, 2003.

23. Le sigle PDV renvoie à l'approche spécifique de la notion de " point de vue » par A. Rabatel. La question n'est plus de savoir comme chez Genette « qui voit » ou « qui sait » mais d'analyser le choix des « moyens linguistiques par lesquels un sujet envisage un objet » (Rabatel $2008: 21$ ).

24. Les recherches d'A. Rabatel peuvent difficilement prendre place dans une partie consacrée aux approches «structuralistes » car c'est précisément parce qu'A. Rabatel s'est éloigné de ces approches en mobilisant des outils relevant d'un cadre «énonciatif » puis « dialogique » qu'il a pu en proposer un renouvellement; ces recherches mériteraient (dans une autre partie) de plus 
amples développements pour être clairement présentées, mais cela nous éloignerait trop de notre propos central.

25. I. Yocaris et D. Zemmour $(2008: 1254)$ ont analysé les variations de profondeur de perspectives spatio-temporelles au sens proposé par A. Rabatel, à partir d'extraits de deux romans de C. Simon. Ils ont donné les exemples suivants : un homme «vu » en même temps à deux moments différents, et donc à la fois comme un homme mur et un vieillard (dédoublement dans le temps) ; deux hommes (souriants sur une photo datant de 1910 et qui ont été tués lors de la guerre de 14-18) décrits comme des « insouciants condamnés à morts " (métalepses figurales), une scène racontée comme pouvant s'être produite une seule fois (scène singulière) ou plusieurs fois (scène itérative), cela grâce au choix des temps verbaux.

26. Précisons que ces récits ont été produits en réponse à la question de savoir si les narrateurs (adolescents habitant le quartier de Harlem à New-York) s'étaient déjà sentis en danger de mort. Tout en reconnaissant la structure de ces récits, W. Labov a surtout insisté sur les aspects rendant «intéressant» le récit pour le narrataire; ces aspects contribuent en partie à la «tension» du récit mais ne s'y réduisent pas.

27. Nous opposons les récits " ponctuels » au récit « global » (« récit des récits ») qui se dégage de l'ensemble de l'entretien (cf. point, cf. infra point 4.1.2

28. Les termes affectifs énoncent « une réaction émotionnelle du sujet parlant en face de cet objet. Dans la mesure où ils impliquent un engagement affectif de l'énonciateur, où ils manifestent sa présence au sein de l'énoncé, ils sont énonciatifs" (Kerbrat-Orecchioni, 1980: 84). Les termes axiologiques sont porteurs d'un jugement de valeur, qu'il soit « péjoratif » ou «mélioratif » (et de ce fait parfois flatteur). Ces deux types de termes sont présentés par Kerbrat-Orecchioni (1980) comme faisant eux-mêmes partie de l'ensemble plus vaste des marques (énonciatives) de la « subjectivité dans le langage ».

29. En fait, Ricœur s'intéresse aux seules productions écrites; mais ses considérations sont à notre avis tout à fait transposables aux productions orales.

30. La mimesis I (temps de la vie) est une préfiguration du champ pratique, elle renvoie à l'expérience pratique qui est à l'origine d'une production discursive. La mimesis II (temps du texte) est une configuration textuelle qui permet une médiation entre les mimesis I et III. La mimesis III (temps de la lecture) est une refiguration par la réception de la production discursive. Elle renvoie à la temporalité de la lecture. Ces trois temporalités peuvent entrer en conflit même si elles présentent des homologies : «Par exemple, un récit est clos, comporte début et fin, ce que "la vie" ne comporte pas ou pas de la même façon et la lecture du texte est entre la clôture du texte et l'ouvert des implicites qu'il suppose ou rend possible » (François, 2004 : 192).

31. Nom d'un grand cabinet d'audit.

32. Il faut savoir que les normes comptables se sont historiquement construites d'abord sur des normes nationales dans des cabinets de moyenne ou petite tailles et sont aujourd'hui bousculées par la «normalisation européenne », laquelle se calque, à quelques nuances près sur les normes anglo-saxonnes des grands cabinets internationaux. Ces derniers ont donc une légitimité forte quant à la mise en pratique des normes à respecter dans l'exercice de la profession (ici les normes comptables).

33. Pour le dire vite, le niveau « conversationnel » ou « dialogal» concerne le dialogue en tant qu'alternance de tours de parole in praesentia tandis que le niveau dialogique englobe le précédent et s'étend au dialogue en tant qu'alternance entre tours de parole in praesentia et in absentia. Les analyses s'inspirant des recherches conversationnelles et/ou ethnométhodologiques anglo-saxonnes (de Sacks à Mondada) ne se décrivent pas comme relevant d'une perspective dialogique, mais peuvent être relues dans cette perspective, en considérant que les reprisesmodifications de son propre discours relèvent d'un auto-dialogisme (cf. 1.1 pour la définition de ce terme). 
34. Notons que certains chercheurs (Schegloff 1968, Roulet 1980) utilisent le terme " conversation " comme un synonyme "d'interaction en face à face ", c'est-à-dire comme un ensemble plus large incluant divers types d'interactions.

35. Notre traduction.

36. Cette perspective ne propose pas d'expression spécifique pour «les récits recueillis lors d'entretien », mais emprunte celles d'«entretien narratif» ou "entretien biographique » (Bertaux, 1997 : 7) aux sociologues.

37. «Style » est ici à prendre dans un sens non littéraire, et correspond à « façon de parler ».

38. L'emploi du terme « sacrifier » est fort pour exprimer ce regret et illustre à quel point ce n'est pas la quantité de termes apparaissant dans un discours qui oriente l'interprétation d'un discours, mais la façon dont certains termes «retentissent» (François 2009) avec plus de force que d'autres sur le récepteur pour exprimer tel ressenti ou telle idée.

39. Notons que l'association du terme de « sacrifice » à " vie de famille » n'est pas faite d'emblée, mais très progressivement, comme s'il s'agissait d'un contenu « délicat » à aborder, difficile à dire : à la 1.3, « sacrifier » est en effet d'abord associé à " certaines choses » tandis qu'est mise en avant «la passion » du métier $(1.1,1.3)$ qui se fait « un peu au détriment d'une vie familiale » (1.6) ; l'association de "sacrifice » et «vie familiale » n'arrive qu'à la 1. 22, dans un troisième mouvement argumentatif qui reprend-modifie les deux précédents.

40. Pour P. Achard, un « registre discursif » correspond à l'hypothèse suivante : certains textes (oraux comme écrits) relevant d'un même « voisinage social » ont des caractéristiques formelles (surtout énonciatives) communes parce qu'ils renvoient à des positionnements idéologiques communs. «Un registre discursif fait correspondre l'énonciateur et le locuteur (place sociale réelle). [...] Nous appellerons genre discursif les discours normalement associés aux registres » (Achard, $1993: 87)$.

41. Au sens où l'ont définie Boutet, Fiala et Simonin-Grumbach (1976) en s'inspirant de la notion de formation discursive (Foucault, 1969). Cette dernière a été définie comme "un ensemble de règles anonymes, historiques, toujours déterminées dans le temps et dans l'espace qui ont défini à une époque donnée, et pour une aire sociale, économique, géographique ou linguistique donnée, les conditions d'exercice de la fonction énonciative» (Foucault, 1969, p. 153) et la formation langagière comme "un ensemble réglé de pratiques langagières, qui organise celles-ci selon des rapports de force en pratiques dominantes et pratiques dominées. Cette notion introduit l'idée qu'il existe des rapports de force entre les pratiques langagières et non seulement que le langagier trace ou reflète des rapports de force extérieurs. [...] Le langage est constitutif, à la fois enjeu et agent, des relations sociales » (Boutet dans Chareaudeau et Maingueneau, 2002 : 272).

\section{ABSTRACTS}

This article begins by explaining the notion of "emotional temporality " (Carcassonne 2004, 2007). Then its indicates to what extent structuralist approaches of the narrative have emphasized a forward-looking temporality (thanks to suspense) and to what extent hermeneutic approaches inspired by Ricœur have emphasized a retrospective temporality (thanks to explanations, justifications and interpretations(performances)). The more recent dialogicalinteractive approach is then underlined to show that it admits the explosion of the narrative structure which it no longer considers as a major criterion to define a narrative. This opens the 
way to exploring new dimensions, in particular that of « emotional temporality ». The article goes on to point out a number of characteristics common to various interviewees in the way of expressing emotional forms of temporality by insisting on the fact that these forms of temporality take shape in the dynamics of the interview (discursive moves) rather than in what is explicitly said. In conclusion, these characteristics are connected with various levels of contexts (of collection, professional, ideological).

Cet article commence par expliquer la notion de «temporalité affective » (Carcassonne 2004, 2007). Puis il indique en quoi les approches structuralistes du récit ont mis l'accent sur une temporalité prospective (grâce au suspens et en quoi les approches herméneutiques inspirées de Ricœur ont mis l'accent sur une temporalité rétrospective (grâce aux explications, justifications et interprétations). Il souligne ensuite que l'approche dialogique-interactive, plus récente, admet l'éclatement de la structure narrative et ne considère plus cette dernière comme un critère incontournable pour définir un récit, ce qui ouvre la voie à l'exploration de nouvelles dimensions, et notamment celle de la « temporalité affective ». L'article pointe ensuite un certain nombre de caractéristiques communes à différents interviewés dans la façon de dire les temporalités affectives en insistant sur le fait que ces temporalités se dessinent dans la dynamique de l'entretien (mouvements discursifs) plus qu'elle ne se disent explicitement. En conclusion, ces caractéristiques sont reliées à différents niveaux de contexte (de recueil, professionnel, idéologique).

\section{INDEX}

Mots-clés: récit, temporalité, entretien, approche dialogique, approche interactive, chronotope, mouvement discursif, signification dessinée, interprétation

Chronological index: XXIe siècle

Geographical index: France

\section{AUTHOR}

\section{MARIE CARCASSONNE}

Université Paris-Dauphine, PSL Research University, CNRS [UMR 7170], Institut de Recherches Interdisciplinaires en Sciences Sociales (IRISSO),75016 Paris, France

Marie Carcassonne est Maîtresse de conférences à l'Université Paris-Dauphine, PSL Research University, CNRS, [UMR 7170], IRISSO. Ses thématiques de recherches s'articulent autour de deux axes principaux : l'un s'intéresse à la didactique et en particulier au rôle du langage dans les apprentissages dans différents types de situations pédagogiques (Ecole, famille, auto-école), l'autre s'intéresse aux processus d'émergence du sens dans différents types d'entretiens narratifs. Elle mobilise dans ces deux axes les notions de « significations dessinées » par les « mouvements discursifs ». Elle a par exemple publié :

- Carcassonne M., 2015, « Analyse discursive de points de vue dans des récits de parcours professionnels extraits d'entretiens », dans Carcassonne M., Cunha D., Donahue C., François F. Rabatel A., 2015, Points de vue sur le point de vue, Limoges, Lambert-Lucas, pp. 77-167 ;

- Carcassonne M., 2012, « Mouvements et places dans un débat scolaire : un travail langagier et identitaire ", dans Sauvage J., Demougin F., (dirs.), dans La construction identitaire à l'école. Perspectives linguistiques et plurielles. Paris, L'Harmattan, p. 45-55. 